\title{
Symposium review: Genetic relationships between different measures of feed efficiency and the implications for dairy cattle selection indexes*
}

\author{
R. J. Tempelman ${ }^{1} \dagger \odot$ and Y. $\mathrm{Lu}^{2}$ \\ ${ }^{1}$ Department of Animal Science, Michigan State University, East Lansing 48824 \\ ${ }^{2}$ Axio Research, a Cytel Company, Seattle, WA 98121
}

\begin{abstract}
A greater number of dairy economic selection indexes are incorporating a measure of feed efficiency (FE) as a key trait. Definitions of FE traits have ranged from dry matter intake (DMI) to residual feed intake (RFI), noting that RFI is effectively DMI adjusted for various energy sink traits such as body weight (BW) and milk energy (MilkE). Other definitions of FE fall between these 2 extremes such as feed saved (FS), which combines RFI and the portion of DMI required to maintain BW. The choice between different FE traits can create confusion as to how to meaningfully compare their heritabilities, estimated breeding values (EBV) and their corresponding reliabilities, and how to differentially incorporate these EBV into selection indexes. If RFI and FS are merely linear functions of DMI, BW, and MilkE with known genetic variances and covariances between these 3 traits, there may be no need to directly compute RFI or FS phenotypes to determine their heritabilities, genetic correlations, EBV, and respective reliabilities for individual animals. We demonstrate how the estimated total genetic merit is invariant to the specification of a FE trait within a selection index. That is, economic weights for a selection index involving one particular FE trait readily convert into the economic weights for a selection index involving a different linear function of that FE trait. We use these different specifications of $\mathrm{FE}$ to provide insight as to the effect of the degree of missingness (e.g., paucity of DMI relative to milk yield records) on the EBV accuracies of the various derivative FE traits. We particularly highlight that the generally observed higher EBV accuracies for DMI, then for FS, and lastly for RFI are partly driven by the greater genetic correlations of DMI with $\mathrm{BW}$

\footnotetext{
Received October 22, 2019.

Accepted February 7, 2020

*Presented as part of the Joint ADSA/Interbull Session: Breeding
} and Genetics Data Pipelines for Implementation of Genomic Evaluation of Novel Traits at the ADSA Annual Meeting, Cincinnati, Ohio, June 2019.

†Corresponding author: tempelma@msu.edu
\end{abstract}

and MilkE and of FS with BW. Finally, we advocate a genetic regression approach to deriving FS and RFI recognizing that genetic versus residual relationships between FE component traits may differ substantially from each other.

Key words: feed efficiency, multiple trait analysis, selection index

\section{INTRODUCTION}

Feed costs have consistently represented nearly half of the variable costs for dairy production. The importance of additional improvements in feed efficiency (FE) has been recently magnified with extended periods of low milk prices and a greater public concern regarding the role of dairy production on climate change via greenhouse gas emissions (de Haas et al., 2017). In turn, climate change may pose future challenges for ensuring consistently high quality and quantity of feedstuffs in some regions (Britt et al., 2018). Expected higher average ambient temperatures over time will also likely increase the proportion of time in which dairy cows experience heat stress beyond their thermoneutral zone, thereby having further implications for feed intake and nutrient requirements of dairy cattle (Hristov et al., 2018).

Genetic selection, augmented by the use of genomic marker information within the last decade (GarciaRuiz et al., 2016), has greatly improved the gross feed efficiency (GFE) of dairy cattle, primarily because of the dilution of maintenance (VandeHaar et al., 2016). The GFE is a ratio measure typically defined as energy captured as milk product divided by the gross energy consumed by the cow. Dilution of maintenance refers to the phenomenon whereby GFE increases naturally with increasing milk production as the proportion of consumed feed used for maintenance should then subsequently decline. Improvements in GFE should contribute to improved economic and environmental sustainability of dairy production as then less animals would be required to produce the same amount of dairy product (Capper and Bauman, 2013). Nevertheless, 
dilution of maintenance becomes increasingly less important with each successive increment in production relative to body size such that it is important to focus on new ways to enhance digestive and metabolic efficiency. In fact, VandeHaar et al. (2016) suggests that feedstuff digestibilities may be declining with higher intakes such that GFE may eventually decline.

Several research initiatives have recognized the importance of collecting feed intakes for genetic evaluations including the Global Dry Matter Initiative (de Haas et al., 2015), the Efficient Dairy Genome Project (https: //genomedairy.ualberta.ca/), an Australian initiative (Pryce et al., 2015), and a USDA-National Institute of Food and Agriculture-funded project (Tempelman et al., 2015). These and other initiatives have led to the development of reference cow populations with both feed intakes and genotypes so that genomic evaluations on various measures of FE can be provided. Nevertheless, there continue to be considerable challenges regarding the genetic evaluation of FE, particularly with respect to a wide array of possible $\mathrm{FE}$ traits to report on such as DMI, residual feed intake (RFI; Kennedy et al., 1993), and more recently, feed saved (FS) as proposed by Pryce et al. (2015). Furthermore, there seems to be substantial variability in the heritabilities and thus reported accuracies of genomic evaluations for different FE traits. For example, de Haas et al. (2015) reported mean genomic accuracies of 0.37 on DMI on reference (genotyped) animals across several countries, each country averaging less than 1,000 genotyped and phenotyped animals per reference population. Pryce et al. (2015) reported mean reliabilities (squared accuracies) of 0.37 for FS and 0.06 for RFI on genotyped bulls based on data generated from a reference population involving less than 1,200 milking cows and growthbased RFI on $<1,000$ growing heifers. Recently, Van Raden et al. (2018b) reported average reliabilities of 0.37 for RFI on cows having DMI phenotypes, and 0.12 for RFI on high net merit young bulls for RFI using the aforementioned USDA-National Institute of Food and Agriculture reference population of nearly 5,000 cows. It is challenging to compare these different scenarios, not only because different populations are involved but also different FE traits, whereas different classes of animals (i.e., cows versus proven bulls versus young bulls) should be expected to differ in reliabilities.

The outline of this review is as follows. First, we intend to provide a clear genetic distinction between the various definitions of $\mathrm{FE}$ traits including DMI, RFI, and FS. We draw these distinctions both from the classical phenotypic regression approach, typically used to derive both RFI and FS, and from a genetic regression approach, extending the presentation of Kennedy et al. (1993) and Lu et al. (2015). These distinctions can be readily gleaned from simple contrasts involving a multiple trait analyses on the key component traits of FE, namely DMI, milk energy (MilkE), and metabolic body weight (MBW). We demonstrate that whichever of these particular FE traits are incorporated within a selection index is completely inconsequential for selection on total genetic merit as suggested earlier by Kennedy et al. (1993) who focused on DMI versus RFI. We further demonstrate how the genetic and residual parameters of these key FE traits are intertwined with each other using simple matrix algebra. We also illustrate the implications of choice of which FE trait to report within a genetic evaluation system on the corresponding reported reliabilities and selection index weights. We conclude with some cautionary notes regarding the implications of heterogeneous relationships between the key component traits of FE across environments at various levels and the interpretation of classical genome-wide association (GWA) inferences involving FE traits.

\section{DISSECTION OF RESIDUAL FEED INTAKE FROM COMPONENT TRAITS}

\section{Phenotypic Residual Feed Intake}

A first realization before debating whether to report or select for DMI versus RFI (e.g., Veerkamp et al., 2013; Berry and Pryce, 2014), for example, should be that RFI is not a directly observable trait; it is merely a statistical construct or a derivative trait. The RFI was first defined by Koch et al. (1963) in the context of efficiency of growth for beef cattle as an estimated residual from regressing DMI, the energy source, on average daily gain and BW, being energy sinks. Within the framework of milk production in dairy cows, a 2-stage model is typically involved (Tempelman et al., 2015). The first stage typically involves fitting DMI as a linear function of various environmental effects (for example, contemporary groups, stage of lactation, and so on), plus partial linear regression coefficients on each of key energy sinks such as MilkE (Mcal) $=9.29 \times \mathrm{kg}$ of fat yield $+5.63 \times \mathrm{kg}$ of protein yield $+3.95 \times \mathrm{kg}$ of lactose yield as per NRC (2001), MBW $=\mathrm{BW}^{0.75}$, and the change in body weight $(\boldsymbol{\Delta} \mathbf{B W})$ in kilograms over a certain specified period of time. In other words, the conceptual model provided in Equation [1] is fitted.

$$
\begin{aligned}
& \mathrm{DMI}=\text { various environmental effects }+b_{1}(\mathrm{MilkE}-\overline{\mathrm{MilkE}}) \\
& +b_{2}(\mathrm{MBW}-\overline{\mathrm{MBW}})+b_{3}(\Delta \mathrm{BW}-\overline{\Delta \mathrm{BW}})+e
\end{aligned}
$$


Note that $b_{1}, b_{2}$, and $b_{3}$ are partial regression coefficients on MilkE, MBW, and $\triangle \mathrm{BW}$, all expressed relative to their mean values $\overline{\mathrm{MilkE}}, \overline{\mathrm{MBW}}$, and $\overline{\Delta \mathrm{BW}}$, respectively, with $e$ being a residual. With all unknown location parameters being fixed effects, one could estimate the corresponding effects using ordinary least squares (OLS). The estimated residual $\hat{e}$ from this model is typically determined to be the RFI "trait" such that, for example, a positive value for $\hat{e}$ implies that the corresponding animal is less efficient than average (i.e., consumes more feed than expected), whereas a negative RFI implies that the animal is above average for efficiency. We subsequently refer to $\hat{e}$ as phenotypic RFI (pRFI) to draw distinctions with genetic RFI as introduced by Kennedy et al. (1993), further extended by $\mathrm{Lu}$ et al. (2015), and discussed later in this paper.

In the second stage model, the pRFI residual is typically modeled as a function of additive genetic effects (a) and potentially other cow-specific effects such as permanent environmental effects $(p e)$ in addition to a second residual $(\varepsilon)$ and an overall mean $(\mu)$.

$$
\mathrm{pRFI}=\hat{e}=\mu+a+p e+\varepsilon .
$$

It is important to add that if repeated measures on pRFI within and across lactations are both available, one might further extend $a$ and pe to account for random regressions on DIM, for example, and partition between lactation $p e$ from within lactation pe (Tempelman et al., 2015; Hurley et al., 2017).

Now if some of the first stage model terms in Equation [1] are known not to be nearly orthogonal to terms in the second stage model in Equation [2] (e.g., nonrandom associations exist between contemporary groups and genetics whereby better managers tend to use superior genetics), then it might be advisable to jointly combine both stages into one model (Tempelman et al., 2015). Furthermore, using Equation [1] to generate pRFI for subsequent analyses using Equation [2] generates heterogeneity of variance for the pRFI response variables based on OLS theory. That is, if $\mathbf{X}_{1}$ denotes the incidence matrix for all classification factors and covariates defined on the right side of Equation [1] and $\hat{\mathbf{e}}=\left\{\hat{e}_{i}\right\}_{i=1}^{n}$ denotes the vector of pRFI "phenotypes" on cows $i=1,2, \ldots, n$, or estimated residuals from fitting Equation [1], then $\operatorname{var}(\hat{\mathbf{e}})=\left(\mathbf{I}_{n}-\mathbf{H}\right) \sigma_{e}^{2}$ using conventional OLS where $\mathbf{H}=\mathbf{X}_{1}\left(\mathbf{X}_{1}^{\prime} \mathbf{X}_{1}\right)^{-1} \mathbf{X}_{1}^{\prime}$ with $\mathbf{H}$ denoting the well-known "hat" or projection matrix, $\mathbf{I}_{n}$ denoting an identity matrix of order $n$, and $\sigma_{e}^{2}$ denoting the residual variance. Again, this residual heteroskedasticity problem would be avoided by combining both stages (Equations [1] and [2]) together into one model.
Per Kennedy et al. (1993), a clearer understanding of the relationship between DMI and pRFI can be best delineated from a multiple trait analysis involving the key base traits being MilkE (trait 1), MBW (trait 2), and DMI (trait 3). We write the genetic merit on these 3 key component traits of $\mathrm{FE}$ for cow $i$ as $\mathbf{u}_{\text {comp }, i}=\left[\begin{array}{lll}u_{M i l k E, i} & u_{M B W, i} & u_{D M I, i}\end{array}\right]^{\prime} ;$ similarly, we represent the corresponding residual vector on cow $i$ in this multiple trait system as $\mathbf{e}_{\text {comp }, i}=\left[\begin{array}{ll}e_{M i l k E, i} e_{M B W, i} & e_{D M I, i}\end{array}\right]^{\prime}$. We subsequently delete the index $i$ in $\mathbf{u}_{c o m p, i}$ and $\mathbf{e}_{c o m p, i}$ and their corresponding elements, assuming momentarily that genetic and residual (co)variances are constant across all animals. We symbolically represent the (co) variance matrices for these 2 vectors as in Equation [3] and use "hat" notation to indicate the corresponding estimates (e.g., $\hat{\sigma}_{u 1}^{2}$ is the estimate of $\sigma_{u 1}^{2}$ ).

$$
\operatorname{var}\left(\mathbf{u}_{c o m p}\right)=\mathbf{G}=\left[\begin{array}{ccc}
\sigma_{u 1}^{2} & \sigma_{u 12} & \sigma_{u 13} \\
\sigma_{u 12} & \sigma_{u 2}^{2} & \sigma_{u 23} \\
\sigma_{u 13} & \sigma_{u 23} & \sigma_{u 3}^{2}
\end{array}\right]
$$

$$
\operatorname{var}\left(\mathbf{e}_{\text {comp }}\right)=\mathbf{R}=\left[\begin{array}{ccc}
\sigma_{e}^{2} & \sigma_{e 12} & \sigma_{e 13} \\
\sigma_{e 12} & \sigma_{e 2}^{2} & \sigma_{e 23} \\
\sigma_{e 13} & \sigma_{e 23} & \sigma_{e 3}^{2}
\end{array}\right]
$$

Ideally, a fourth trait ( $\Delta \mathrm{BW})$ would also be included in this multiple trait system, but Lu et al. (2015) faced considerable challenges with estimation and convergence with the corresponding 4-trait mixed model, partly because of the near-zero heritability estimate of $\triangle \mathrm{BW}$. Hence, their multiple trait model, as well as those in Lu et al. $(2017,2018)$, simply included $\Delta \mathrm{BW}$ as a covariate for DMI. Furthermore, it has also been contended that the partial DMI relationship on a positive $\triangle \mathrm{BW}$ should be modeled separately from that on a negative $\Delta \mathrm{BW}$ (Berry and Crowley, 2013).

To help illustrate the genetic and residual relationships between DMI and pRFI, we refer back to the analyses conducted by Lu et al. (2018) involving 28-d records on DMI, MilkE, and MBW taken on 6,937 cows recorded between 50 and 200 DIM. We use their estimated genetic $(\hat{\mathbf{G}})$ and residual $(\hat{\mathbf{R}})$ variance covariance matrices as provided in Equation [4].

$$
\hat{\mathbf{G}}=\left[\begin{array}{ccc}
2.69 & 0.14 & 1.10 \\
0.14 & 26.42 & 2.61 \\
1.10 & 2.61 & 1.08
\end{array}\right] ; \hat{\mathbf{R}}=\left[\begin{array}{ccc}
9.50 & 4.27 & 3.36 \\
4.27 & 31.98 & 4.18 \\
3.36 & 4.18 & 3.52
\end{array}\right] . \quad[4]
$$


For additional insights, we further reparameterize $\hat{\mathbf{G}}$ and $\hat{\mathbf{R}}$ as genetic $\left(\hat{\boldsymbol{\rho}}_{G}\right)$ and residual correlation $\left(\hat{\boldsymbol{\rho}}_{R}\right)$ matrices in Equation [5]:

$$
\hat{\boldsymbol{\rho}}_{G}=\left[\begin{array}{ccc}
1 & 0.02 & 0.64 \\
0.02 & 1 & 0.48 \\
0.64 & 0.48 & 1
\end{array}\right] ; \hat{\boldsymbol{\rho}}_{R}=\left[\begin{array}{ccc}
1 & 0.25 & 0.58 \\
0.25 & 1 & 0.39 \\
0.58 & 0.39 & 1
\end{array}\right] .
$$

Note, for example, that the genetic correlation between the 2 energy sink traits MilkE (trait 1) and MBW (trait 2 ) is near zero, confirming results consistently observed in previous studies (Berry and Crowley, 2013; Manzanilla-Pech et al., 2016), whereas the corresponding estimated residual correlation (0.25) is relatively moderate. Furthermore, both the genetic and residual correlations between DMI (trait 3) with the 2 energy sink traits MilkE and MBW (traits 1 and 2) are relatively large $(0.39-0.64)$. This is not surprising as greater production and maintenance costs can only be sustained with greater DMI on both genetic and nongenetic levels. From the diagonal elements of $\hat{\mathbf{G}}$ and $\hat{\mathbf{R}}$ in Equation [4], it can be readily determined that the estimated heritabilities for the 3 traits are $\hat{h}_{M i l k E}^{2}=0.22, \hat{h}_{M B W}^{2}=0.45$, and $\hat{h}_{D M I}^{2}=0.23$.

Consider the phenotypic variance-covariance matrix $(\hat{\mathbf{P}}=\hat{\mathbf{G}}+\hat{\mathbf{R}})$ based on summing together the 2 matrices in Equation [4]. We further partition $\hat{\mathbf{P}}$ into various subblocks due to energy sink and source (co)variance matrices $\hat{\mathbf{P}}_{\text {sink,sink }}, \hat{\mathbf{P}}_{\text {sink,source }}=\hat{\mathbf{P}}_{\text {source,sink }}^{\prime}$, and $\hat{\mathbf{P}}_{\text {source,source }}$ as illustrated by the line boundaries in Equation [6].

$\hat{\mathbf{P}}=\hat{\mathbf{G}}+\hat{\mathbf{R}}=\left[\begin{array}{ccc}12.19 & 4.41 & 4.45 \\ 4.41 & 58.40 & 6.79 \\ 4.45 & 6.79 & 4.59\end{array}\right]=\left[\begin{array}{cc}\hat{\mathbf{P}}_{\text {sink, sink }} & \hat{\mathbf{P}}_{\text {sink,source }} \\ \hat{\mathbf{P}}_{\text {source, sink }} & \hat{\mathbf{P}}_{\text {source,source }}\end{array}\right]$

Borrowing from Kennedy et al. (1993), one could readily derive the partial regression coefficients $b_{1}$ and $b_{2}$ using conditional multivariate normal theory in Equation [7] below, instead of using the OLS approach illustrated in Equation [1].

$$
\begin{aligned}
& {\left[\begin{array}{l}
\hat{b}_{1} \\
\hat{b}_{2}
\end{array}\right]=\hat{\mathbf{P}}_{\text {source,sink }}\left(\hat{\mathbf{P}}_{\text {sink,sink }}\right)^{-1}=} \\
& {\left[\begin{array}{ll}
4.45 & 6.79
\end{array}\right]\left[\begin{array}{cc}
12.19 & 4.41 \\
4.41 & 58.40
\end{array}\right]^{-1}=\left[\begin{array}{l}
0.33 \mathrm{Mcal} / \mathrm{kg} \\
0.09 \mathrm{~kg}^{0.75} / \mathrm{kg}
\end{array}\right] .}
\end{aligned}
$$

These estimates in Equation [7] are the same as those reported by $\mathrm{Lu}$ et al. (2018) even though they used the more conventional partial regression approach from combining Equations [1] and [2] together within one single stage mixed effects model. However, the approach taken in Equation [7] is recommended in that it allows for missing records on any of the 3 traits. Furthermore, it mitigates the potential effect of measurement error on the covariate traits (MilkE and MBW), which can badly distort partial regression parameter estimates in a linear model analysis (Carroll et al., 2006).

\section{Insights from Multiple Trait Analyses}

Conditional on the estimated variance components $\hat{\mathbf{G}}$ and $\hat{\mathbf{R}}$, one can readily compute the EBV for the 3 traits on any animal using multiple trait mixed model equations. Noting that the estimated partial regression coefficients $\hat{b}_{1}$ and $\hat{b}_{2}$ define the partial phenotypic relationships of DMI with MilkE and MBW that are used to determine pRFI, these coefficients also subsequently define the genetic and residual relationships between the same traits as also illustrated by Kennedy et al. (1993). In other words, the genetic merit for pRFI can be readily determined as $u_{p R F I}=\mathbf{k}_{p R F I}^{\prime} \mathbf{u}_{c o m p}$, where $\mathbf{k}_{p R F I}^{\prime}=\left[\begin{array}{lll}-\hat{b}_{1} & -\hat{b}_{2} & 1\end{array}\right]$. Suppose that one wishes to estimate the joint genetic variance-covariance matrix $\widehat{\operatorname{var}}\left(\boldsymbol{u}_{p}\right)$ of $\mathbf{u}_{p}=\left[\begin{array}{llll}u_{\text {MilkE }} & u_{M B W} & u_{D M I} & u_{p R F I}\end{array}\right]^{\prime}$. Instead of conducting a multiple trait analysis involving any or all of these 4 traits as has been done previously (e.g., Veerkamp et al., 1995; Manzanilla-Pech et al., 2016), one could simply use $\hat{\mathbf{G}}$ to compute $\widehat{\operatorname{var}}\left(\mathbf{u}_{p}\right)=\mathbf{K}_{p} \hat{\mathbf{G}} \mathbf{K}_{p}^{\prime}$ with $\mathbf{K}_{p}^{\prime}=\left[\begin{array}{ll}\mathbf{I}_{3} & \mathbf{k}_{p R F I}\end{array}\right]$ for $\mathbf{I}_{3}$ being an identity matrix of order 3 . The estimated covariance matrix $\widehat{\operatorname{var}}\left(\boldsymbol{u}_{p}\right)$ and the corresponding correlation matrix $\hat{\boldsymbol{\rho}}\left(\mathbf{u}_{p}\right)$ are provided in Equation [8].

$$
\begin{gathered}
\widehat{\operatorname{var}}\left(\boldsymbol{u}_{p}\right)=\widehat{\operatorname{var}}\left(\left[\begin{array}{c}
u_{M i l k E} \\
u_{M B W} \\
u_{D M I} \\
u_{p R F I}
\end{array}\right]\right)=\left[\begin{array}{cccc}
2.69 & 0.14 & 1.10 & 0.19 \\
0.14 & 26.42 & 2.61 & 0.15 \\
1.10 & 2.61 & 1.08 & 0.48 \\
0.19 & 0.15 & 0.48 & 0.40
\end{array}\right] ; \\
\hat{\boldsymbol{\rho}}\left(\mathbf{u}_{p}\right)=\left[\begin{array}{cccc}
1 & 0.02 & 0.64 & 0.18 \\
0.02 & 1 & 0.48 & 0.05 \\
0.64 & 0.48 & 1 & 0.73 \\
0.18 & 0.05 & 0.73 & 1
\end{array}\right] .
\end{gathered}
$$


Note, for example, that the estimated genetic covariance between DMI and pRFI can be readily determined as $\hat{\sigma}_{u_{3}}^{2}-\hat{b}_{1} \hat{\sigma}_{u_{13}}-\hat{b}_{2} \hat{\sigma}_{u_{23}}=1.08-0.33(1.10)-0.09(2.61)$ $=0.48$ [i.e., the $[3,4]$ element of $\widehat{\operatorname{var}}\left(\boldsymbol{u}_{p}\right)$ ], whereas the estimated genetic variance of $\mathrm{pRFI}$ is $\mathbf{k}_{p R F I}^{\prime} \hat{\mathbf{G}} \mathbf{k}_{p R F I}=$ 0.40 .

Similarly, one can determine the estimated residual variance-covariance matrix $\widehat{\operatorname{var}}\left(\mathbf{e}_{p}\right)$ with $\mathbf{e}_{p}=\left[\begin{array}{llll}e_{\text {MilkE }} & e_{M B W} & e_{D M I} & e_{p R F I}\end{array}\right]^{\prime}$ for the 4 traits using $\widehat{\operatorname{var}}\left(\mathbf{e}_{p}\right)=\mathbf{K}_{p} \hat{\mathbf{R}} \mathbf{K}_{p}^{\prime}$. Here $\widehat{\operatorname{var}}\left(\mathbf{e}_{p}\right)$ and its corresponding correlation matrix $\hat{\boldsymbol{\rho}}\left(\mathbf{e}_{p}\right)$ are provided in Equation [9].

$$
\begin{gathered}
\widehat{\operatorname{var}}\left(\mathbf{e}_{p}\right)=\widehat{\operatorname{var}}\left(\left[\begin{array}{l}
e_{M i l k E} \\
e_{M B W} \\
e_{D M I} \\
e_{p R F I}
\end{array}\right]\right)=\left[\begin{array}{cccc}
9.50 & 4.27 & 3.36 & -0.19 \\
4.27 & 31.98 & 4.18 & -0.15 \\
3.36 & 4.18 & 3.52 & 2.02 \\
-0.19 & -0.15 & 2.02 & 2.10
\end{array}\right] ; \\
\hat{\boldsymbol{\rho}}\left(\mathbf{e}_{p}\right)=\left[\begin{array}{cccc}
1 & 0.25 & 0.58 & -0.04 \\
0.25 & 1 & 0.39 & -0.02 \\
0.58 & 0.39 & 1 & 0.74 \\
-0.04 & -0.02 & 0.74 & 1
\end{array}\right] .
\end{gathered}
$$

Note again that the estimated residual variance of pRFI can be determined as $\mathbf{k}_{p R F I}^{\prime} \mathbf{R k}_{p R F I}=2.10$; that is, the $[4,4]$ element of $\widehat{\operatorname{var}}\left(\mathbf{e}_{p}\right)$. Thus, the estimated heritability of pRFI is then simply $\hat{h}_{p R F I}^{2}=\mathbf{k}_{p R F I}^{\prime} \hat{\mathbf{G}} \mathbf{k}_{p R F I} /\left(\mathbf{k}_{p R F I}^{\prime} \hat{\mathbf{G}} \mathbf{k}_{p R F I}+\mathbf{k}_{p R F I}^{\prime} \hat{\mathbf{R}} \mathbf{k}_{p R F I}\right)=$ $0.40 /(0.40+2.10)=0.16$. Hence $\hat{h}_{p R F I}^{2}$ is a function of every single element in $\hat{\mathbf{G}}$ and $\hat{\mathbf{R}}$ involving each of the 3 key component traits of FE. This same $\hat{h}_{p R F I}^{2}$ estimate was also reported by $\mathrm{Lu}$ et al. (2018), albeit using a classical pRFI analysis in which DMI was explicitly regressed on MilkE and MBW within a single step model combining Equations [1] and [2] above. Given that $\hat{b}_{1}$ and $\hat{b}_{2}$ as used within $\mathbf{k}_{p R F I}^{\prime}$ are also functions of estimated phenotypic (and hence genetic and residual) variances and covariances, the heritability of pRFI is a particularly complex function of $\mathbf{G}$ and $\mathbf{R}$. In fact, Kennedy et al. (1993) concluded that $\hat{h}_{p R F I}^{2}$ is particularly sensitive to variation in the residual correlations between energy source and sink traits. Note that estimated residual covariances between pRFI with MBW and with MilkE (Equation [9]) have the same absolute values, albeit of opposite signs, as the corresponding genetic covariance estimates (Equation [8]). Hence their sum is the corresponding phenotypic covariances, which are constrained to be equal to zero as per Kennedy et al. (1993).

\section{Genetic Regressions}

Recognizing the complications that associate with pRFI (i.e., not necessarily genetically independent of MilkE or MBW, heritability being sensitive to residual covariances between traits), Kennedy et al. (1993) derived genetic RFI (gRFI) as a FE trait that is genetically independent of MilkE and MBW. We would add a key additional concern with the use of pRFI to those expressed by Kennedy et al. (1993). That is, the genetic relationships (i.e., partial regression coefficients) between the component traits of FE can differ substantially from the corresponding residual relationships such that estimated partial regression coefficients used to derive pRFI are not readily interpretable. That is, the estimates of $b_{1}$ or $b_{2}$ used to derive pRFI may be highly sensitive to population or familial structure.

The need to distinguish differences in relationships between traits at different levels (i.e., within clusters as opposed to across clusters) has been referred to Simpson's paradox (Wagner, 1982) or ecological fallacy (Robinson, 1950). A clear nongenetic example in dairy cattle involves work by Bello et al. (2012) who demonstrated that cow-level (i.e., within-herd) relationships between milk production and reproductive performance may differ substantially from the herd-level (i.e., across-herd) relationships. Specifically, Bello et al. (2012) inferred associations to be far more antagonistic within herds (i.e., higher producing cows having lower reproductive performance) compared with across herds (i.e., higher producing herds do not generally have lower than average reproductive performance); furthermore, they cited several other examples in dairy science where ecological fallacies were noted. In such cases, one single measure of a partial regression or correlation relationship between traits might be virtually meaningless. For similar reasons, phenotypic regression models to derive pRFI (i.e., Equations [1] or Equation [7]) should be discouraged if genetic relationships between DMI with MilkE and MBW are potentially different from residual relationships as, again, inferences on $b_{1}$ and $b_{2}$ could be very sensitive to population structure.

Lu et al. (2015) took a slightly different approach to Kennedy et al. (1993) in determining gRFI. Lu et al. (2015) reparameterized the multiple trait mixed model equations using the Cholesky decomposition to develop a recursive relationship between the component traits of $\mathrm{FE}$ at both the genetic and at the residual level as illustrated in Equations [10a], [10b], and [10c].

$$
\begin{gathered}
u_{1 i}=u_{1 i} ; \quad e_{1 i}=e_{1 i}, \\
u_{2 i}=a_{1}^{(u)} u_{1 i}+u_{2 \mid 1 i} ; \quad e_{2 i}=a_{1}^{(e)} e_{1 i}+e_{2 \mid 1 i},
\end{gathered}
$$


$u_{3 i}=b_{1}^{(u)} u_{1 i}+b_{2}^{(u)} u_{2 i}+u_{31,2 i} ; \quad e_{3 i}=b_{1}^{(e)} e_{1 i}+b_{2}^{(e)} e_{2 i}+e_{3 \mid 1,2 i}$.

$[10 \mathrm{c}]$

For example, in the order provided, MilkE (i.e., trait or subscript 1) is specified first per Equation [10a]. Then MBW (trait 2) is specified conditionally on MilkE in Equation [10b] such that $u_{2 \mid 1 i}=u_{2 i}-a_{1}^{(u)} u_{1 i}$ and $e_{211 i}=e_{2 i}-a_{1}^{(e)} e_{1 i}$ pertain to the genetic and residual effects of MBW adjusted for MilkE on cow $i$. Finally, Equation [10c] provides the conditioning of DMI (trait 3) on MilkE and MBW leading to the genetic and residual effects of gRFI as

$$
u_{g R F I, i} \equiv u_{3 \mid 1,2 i}=u_{3 i}-b_{1}^{(u)} u_{1 i}+b_{2}^{(u)} u_{2 i}
$$

and

$$
e_{g R F I, i} \equiv e_{3 \mid 1,2 i}=e_{3 i}-b_{1}^{(e)} e_{1 i}+b_{2}^{(e)} e_{2 i},
$$

respectively. This recursive strategy is not much unlike how Quaas (1976) used the Cholesky decomposition on the numerator relationship matrix to demonstrate recursive relationships between the genetic merits of parents and offspring.

Now, $a_{1}^{(u)}, b_{1}^{(u)}$, and $b_{2}^{(u)}$ are partial regression coefficients on the genetic level, whereas $a_{1}^{(e)}, b_{1}^{(e)}$, and $b_{2}^{(e)}$ are partial regression coefficients on the residual level. One could adapt Equation [7] but using $\hat{\mathbf{G}}$ from Equation [4] instead of $\hat{\mathbf{P}}$, as also illustrated by Kennedy et al. (1993), to estimate $b_{1}^{(u)}$ and $b_{2}^{(u)}$ as per Equation [11].

$$
\begin{aligned}
& {\left[\begin{array}{l}
\hat{b}_{1}^{(u)} \\
\hat{b}_{2}^{(u)}
\end{array}\right]=\hat{\mathbf{G}}_{\text {source,sink }}\left(\hat{\mathbf{G}}_{\text {sink,sink }}\right)^{-1}} \\
& =\left[\begin{array}{ll}
1.10 & 2.61
\end{array}\right]\left[\begin{array}{cc}
2.69 & 0.14 \\
0.14 & 26.42
\end{array}\right]^{-1}=\left[\begin{array}{c}
0.40 \mathrm{~kg} / \mathrm{Mcal} \\
0.097 \mathrm{~kg} / \mathrm{kg}^{0.75}
\end{array}\right] .
\end{aligned}
$$

Similarly, one could derive the estimates of the residual coefficients $b_{1}^{(e)}$ and $b_{2}^{(e)}$ in a similar manner using submatrices of $\hat{\mathbf{R}}$ as per Equation [12].

$$
\begin{aligned}
& {\left[\begin{array}{l}
\hat{b}_{1}^{(e)} \\
\hat{b}_{2}^{(e)}
\end{array}\right]=\hat{\mathbf{R}}_{\text {source,sink }}\left(\hat{\mathbf{R}}_{\text {sink,sink }}\right)^{-1}} \\
& =\left[\begin{array}{ll}
3.36 & 4.18
\end{array}\right]\left[\begin{array}{cc}
9.50 & 4.27 \\
4.27 & 31.98
\end{array}\right]^{-1}=\left[\begin{array}{c}
0.31 \mathrm{~kg} / \mathrm{Mcal} \\
0.089 \mathrm{~kg} / \mathrm{kg}^{0.75}
\end{array}\right]
\end{aligned}
$$

Note that the estimated genetic partial regression coefficients $\hat{b}_{1}^{(u)}$ and $\hat{b}_{2}^{(u)}$ are slightly higher than the corre- sponding estimated residual coefficients $\hat{b}_{1}^{(e)}$ and $\hat{b}_{2}^{(e)}$, whereas the estimated phenotypic coefficients $\hat{b}_{1}$ and $\hat{b}_{2}$ from Equation [7] lie in between, as might be anticipated. Similarly, Lu et al. (2017) inferring station-specific differences in these coefficients determined that $b_{1}^{(u)}$ and $b_{2}^{(u)}$ were estimated to be consistently higher than the corresponding $b_{1}^{(e)}$ and $b_{2}^{(e)}$. Lu et al. (2015) analytically demonstrated that if partial regressions are the same at both the genetic and the residual level (i.e., $b_{1}^{(u)}$ $=b_{1}^{(e)}$ and $\left.b_{2}^{(u)}=b_{2}^{(e)}\right)$, then there is no distinction between pRFI and gRFI (i.e., pRFI $\equiv$ gRFI).

Again, starting with a multiple trait mixed model analysis on the $3 \mathrm{FE}$ component traits, the genetic merit for gRFI can be determined as $u_{g R F I}=\mathbf{k}_{g R F I(u)}^{\prime} \mathbf{u}_{\text {comp }}$, where $\quad \mathbf{k}_{g R F I(u)}^{\prime}=\left[\begin{array}{lll}\hat{b}_{1}^{(u)} & -\hat{b}_{2}^{(u)} & 1\end{array}\right] . \quad$ Define $\mathbf{u}_{g}=\left[\begin{array}{llll}u_{\text {MilkE }} & u_{M B W} & u_{D M I} & u_{g R F I}\end{array}\right]^{\prime}$. As demonstrated previously for pRFI, one could simply determine the estimated genetic variance covariance matrix for gRFI with component traits using $\widehat{\operatorname{var}}\left(\mathbf{u}_{g}\right)=\mathbf{K}_{u} \hat{\mathbf{G}} \mathbf{K}_{u}^{\prime}$ with $\mathbf{K}_{u}^{\prime}=\left[\begin{array}{ll}\mathbf{I}_{3} & \mathbf{k}_{g R F I}\end{array}\right]$. The corresponding estimated genetic covariance $\left[\widehat{\operatorname{var}}\left(\mathbf{u}_{g}\right)\right]$ and correlation $\left[\hat{\rho}\left(\mathbf{u}_{g}\right)\right]$ matrices are provided in Equation [13].

$$
\begin{gathered}
\widehat{\operatorname{var}}\left(\mathbf{u}_{g}\right)=\widehat{\operatorname{var}}\left(\left[\begin{array}{l}
u_{M i l k E} \\
u_{M B W} \\
u_{D M I} \\
u_{p R F I}
\end{array}\right]\right)=\left[\begin{array}{cccc}
2.69 & 0.14 & 1.10 & 0 \\
0.14 & 26.42 & 2.61 & 0 \\
1.10 & 2.61 & 1.08 & 0.38 \\
0 & 0 & 0.38 & 0.38
\end{array}\right] ; \\
\hat{\rho}\left(\mathbf{u}_{g}\right)=\left[\begin{array}{cccc}
1 & 0.02 & 0.64 & 0 \\
0.02 & 1 & 0.48 & 0 \\
0.64 & 0.48 & 1 & 0.60 \\
0 & 0 & 0.60 & 1
\end{array}\right] .
\end{gathered}
$$

Notice in Equation [13] that, by definition (Kennedy et al., 1993), the genetic correlation between gRFI with MilkE and with MBW are both estimated to be 0 . Furthermore, the genetic covariance between DMI and gRFI and the genetic variance of gRFI are both estimated to be $\hat{\sigma}_{u_{g R F I}}^{2} \equiv \hat{\sigma}_{u_{3 \mid 1,2}}^{2}=\hat{\sigma}_{u_{3}}^{2}-\hat{b}_{1}^{(u)} \hat{\sigma}_{u_{13}}-\hat{b}_{2}^{(u)} \hat{\sigma}_{u_{23}}=$ $1.08-0.40(1.10)-0.097(2.61)=0.38$ as per Lu et al. (2018) and readily apparent from the $[3,4]$ and $[4,4]$ elements of $\widehat{\operatorname{var}}\left(\mathbf{u}_{g}\right)$. Hence the genetic correlation between DMI and gRFI can be readily derived as $\operatorname{corr}\left(u_{D M I}\right.$, $\left.u_{g R F I}\right)=\sqrt{\sigma_{u, g R F I}^{2} / \sigma_{u, D M I}^{2}}$ estimated here to be $\sqrt{0.38 / 1.08}$ $=0.60$ as per Lu et al. (2018) and provided as the [3,4] element of $\hat{\rho}\left(\mathbf{u}_{g}\right)$ in Equation [13]. One can similarly 
derive the corresponding residual variance-covariance matrix, albeit using a different contrast vector than that used to determine $u_{g R F I}$ (i.e., $e_{g R F I}=\mathbf{k}_{g R F I(e)}^{\prime} \mathbf{e}_{\text {comp }}$, where $\left.\mathbf{k}_{g R F I(e)}^{\prime}=\left[\begin{array}{lll}\hat{b}_{1}^{(e)} & -\hat{b}_{2}^{(e)} & 1\end{array}\right]\right)$. Define

$$
\mathbf{e}_{g}=\left[\begin{array}{llll}
e_{M i k E} & e_{M B W} & e_{D M I} & e_{g R F I}
\end{array}\right]^{\prime} .
$$

Hence the required estimated residual variance covariance matrix is $\widehat{\operatorname{var}}\left(\mathbf{e}_{g}\right)=\mathbf{K}_{e} \hat{\mathbf{R}} \mathbf{K}_{e}^{\prime}$ with $\mathbf{K}_{e}^{\prime}=\left[\begin{array}{ll}\mathbf{I}_{3} & \mathbf{k}_{g R F I(e)}\end{array}\right]$. The estimated residual covariance matrix $\left[\widehat{\operatorname{var}}\left(\mathbf{e}_{g}\right)\right]$ and corresponding correlation matrix $\left[\hat{\rho}\left(\mathbf{e}_{g}\right)\right]$ are provided in Equation [14].

$$
\begin{gathered}
\widehat{\operatorname{var}}\left(\mathbf{e}_{g}\right)=\widehat{\operatorname{var}}\left[\begin{array}{c}
e_{M i l k E} \\
e_{M B W} \\
e_{D M I} \\
e_{g R F I}
\end{array}\right]=\left[\begin{array}{cccc}
9.50 & 4.27 & 3.36 & 0 \\
4.27 & 31.98 & 4.18 & 0 \\
3.36 & 4.18 & 3.52 & 2.09 \\
0 & 0 & 2.09 & 2.09
\end{array}\right] ; \\
\hat{\rho}\left(\mathbf{e}_{g}\right)=\left[\begin{array}{cccc}
1 & 0.25 & 0.58 & 0 \\
0.25 & 1 & 0.39 & 0 \\
0.58 & 0.39 & 1 & 0.77 \\
0 & 0 & 0.77 & 1
\end{array}\right] .
\end{gathered}
$$

Note that the residual covariance between DMI and gRFI defined as $\sigma_{e_{g R F I}}^{2}$ is estimated to be 2.09, being the $[3,4]$ and $[4,4]$ elements of $\widehat{\operatorname{var}}\left(\mathbf{e}_{g}\right)$, whereas the residual correlation between DMI and gRFI is defined as $\operatorname{corr}\left(e_{D M I}, e_{g R F I}\right)=\sqrt{\sigma_{e, g R F I}^{2} / \sigma_{e, D M I}^{2}}$ (Lu et al., 2018) and estimated to be $\sqrt{2.09 / 3.52}=0.77$ as provided in the $[3,4]$ element of $\hat{\rho}\left(\mathbf{e}_{g}\right)$ in Equation [14].

One can readily use the specifications of $\operatorname{corr}\left(u_{D M I}\right.$, $\left.u_{g R F I}\right)$ and $\operatorname{corr}\left(e_{D M I}, e_{g R F I}\right)$ from Lu et al. (2018) to determine that $h_{D M I}^{2}>h_{g R F I}^{2}$ only if $\operatorname{corr}\left(e_{D M I}, e_{g R F I}\right)>$ $\operatorname{corr}\left(u_{D M I}, u_{g R F I}\right)$ or equivalently if $\sigma_{e, g R F I}^{2} / \sigma_{e, D M I}^{2}>$ $\sigma_{u, g R F I}^{2} / \sigma_{u, D M I}^{2}$. In other words, if the proportion of the residual variance of DMI due to gRFI exceeds the corresponding proportion of the genetic variance of DMI due to gRFI, then the heritability of gRFI will be less than that of DMI. Lower heritability estimates are typically observed for pRFI relative to DMI (Berry and Crowley, 2013), which indirectly suggests that $\sigma_{e, g R F I}^{2} / \sigma_{e, D M I}^{2}>\sigma_{u, g R F I}^{2} / \sigma_{u, D M I}^{2}$. Hence, with all other things being equal, a decrease in measurement error on DMI with, say, more stringent feed DM assessment protocols (Seymour et al., 2019) should increase $h_{D M I}^{2}$. However, $h_{g R F I}^{2}$, and hence likely $h_{p R F I}^{2}$, would continue to be less than $h_{D M I}^{2}$ given that there would likely be even greater divergence between $\sigma_{e, g R F I}^{2} / \sigma_{e, D M I}^{2}$ and $\sigma_{u, g R F I}^{2} / \sigma_{u, D M I}^{2}$.

Note that the multiple trait model approach ensures that MilkE and MBW are both genetically and residually independent of gRFI as indicated by the zeroes present in the $[1,4]$ and $[2,4]$ elements of both $\widehat{\operatorname{var}}\left(\mathbf{u}_{g}\right)$ and $\widehat{\operatorname{var}}\left(\mathbf{e}_{g}\right)$. This is somewhat different from what was presented for residual effects in Kennedy et al. (1993) who defined the residual term $e_{g R F I}$ for gRFI using the same contrast vector $\mathbf{k}_{g R F I(u)}^{\prime}$ defining genetic relationships between DMI and the energy sink traits.

Practically speaking, pRFI may not be too different from gRFI if the partial regression coefficients at the genetic versus residual level (and hence then at the phenotypic level too) are not vastly different (i.e., $b_{1}^{(u)} \approx b_{1}^{(e)}$ and $b_{2}^{(u)} \approx b_{2}^{(e)}$. The contrast matrix $\mathbf{K}_{R F I}=$ $\left[\mathbf{k}_{p R F I} \mathbf{k}_{g R F I(u)}\right]^{\prime}$ can be used to define the joint genetic merit of pRFI and gRFI as a function of the FE component traits. Hence, the joint genetic effects of the 2 traits is $\mathbf{K}_{R F I} \mathbf{u}_{\text {comp }}$ with variance covariance matrix $\mathbf{K}_{R F I} \mathbf{G K}_{R F I}^{\prime}$. Using $\mathbf{G}=\hat{\mathbf{G}}$ as provided previously, the estimated genetic covariance between pRFI and gRFI is 0.38 , based on the off-diagonal of $\mathbf{K}_{R F I} \hat{\mathbf{G}} \mathbf{K}_{R F I}^{\prime}$, with a corresponding estimated genetic correlation of 0.98 . If $b_{1}^{(u)} \approx b_{1}^{(e)}$ and $b_{2}^{(u)} \approx b_{2}^{(e)}$, it may be advisable to work with pRFI to allow statistically more efficient inferences on a single set of partial regression coefficients $b_{1}$ and $b_{2}$ rather than separate coefficients specified at the genetic and residual levels.

Borrowing from Lu et al. (2018), one can readily determine the contributions of gRFI and the energy sink traits to the genetic effects of DMI as in Equation [15]:

$$
\begin{aligned}
& \hat{\sigma}_{u_{D M I}}^{2}= \\
& \left(\hat{b}_{1}^{(u)}\right)^{2} \hat{\sigma}_{u_{\text {MikE }}}^{2}+\left(\hat{b}_{2}^{(u)}\right)^{2} \hat{\sigma}_{u_{M B W}}^{2}+2\left(\hat{b}_{1}^{(u)} \hat{b}_{2}^{(u)}\right)_{\sigma_{u_{M i k E, M B W}}^{2}}+\hat{\sigma}_{u_{g R F I}}^{2} \\
& =(0.4030)^{2} 2.69+(0.0966)^{2} 26.42+2(0.4030)(0.0966) 0.14+0.38 \\
& =0.44+0.25+0.01+0.38 \\
& =1.08
\end{aligned}
$$

In other words, $41 \%(0.44 / 1.08)$ of the genetic variability of DMI is estimated to be due to the genetic effects of MilkE with another $23 \%(0.25 / 1.08)$ due to MBW and less than 1\% (0.01/1.08) due to the genetic covariance between MilkE and MBW. In other words, nearly two-thirds of the total genetic variation in DMI can be explained by MilkE and MBW. The remaining proportion, $35 \%(0.38 / 1.08)$, is due to RFI or the "unexplained" genetic variation of DMI which, again, also 
defines the squared genetic correlation between these 2 "traits."

\section{OTHER FEED EFFICIENCY TRAITS}

\section{Feed Saved}

The FS, now more frequently referred to as "saved feed" (Pellikaan, 2018; Lidauer et al., 2019), was proposed by Pryce et al. (2015) as an alternative FE measure to RFI. The FS includes not only the metabolic efficiency component of RFI but also the effect of $\mathrm{BW}$ on feed intake as required for maintenance. Feed costs should be decreased by additionally selecting for smaller cows (i.e., recall from Equation [15] that nearly $1 / 4$ of the genetic variation in DMI is due to MBW). Hence, FS may be more attractive than pRFI or gRFI as a trait for FE selection in that it targets cows that have the genetic propensity to use or "save" a greater proportion of their DMI for milk production. Again, this ability may be because of superior metabolic efficiency (i.e., lower RFI), lower maintenance costs (i.e., smaller MBW), or both. Nevertheless, given that RFI is more narrowly defined, it is of likely greater interest than FS with respect to biological inferences such as GWA analysis to identify genomic regions or genes associated with FE. That is, FS may be too complex to interrogate for a GWA study, given that it involves both a combination of metabolic efficiency and body size.

Now Pryce et al. (2015) derived FS with respect to a lifetime contribution or scale. The FE measures intended for selection, as with all economically important traits, should preferably be determined on a scale encapsulating a cow's entire lifetime, to fit within a selection index like the Net Merit Index in the United States (Van Raden et al., 2018a). Admittedly, this can be difficult to determine such that some assumptions need to be made. For example, FE on a lifetime basis should account for body tissue gain and the feed consumed as a heifer and as a dry cow, which may account for between 15 and $30 \%$ of the feed that a cow eats during her lifetime (VandeHaar et al., 2016); of course, this proportion could vary between cows just because of variability in reproductive performance. For illustrative purposes only, we will focus trait definitions on the scale of daily data within 50 to 200 DIM per Lu et al. (2015) in this review.

Suppose that DMI is preadjusted for all environmental covariates and for $\Delta \mathrm{BW}$ in Equation [1] to create $\mathrm{DMI}_{\text {adj }}$ for further use in Equation [16].

$\mathrm{DMI}_{\mathrm{adj}}=b_{1}(\mathrm{MilkE}-\overline{\mathrm{MilkE}})+b_{2}(\mathrm{MBW}-\overline{\mathrm{MBW}})+\mathrm{pRFI}$.
One can combine the last 2 terms in Equation [16] and multiply that sum by -1 to be phenotypic FS (pFS) per Pryce et al. (2015) and as in Equation [17].

$$
\begin{aligned}
& \mathrm{FS}=-1\left[b_{2}(\mathrm{MBW}-\overline{\mathrm{MBW}})+\mathrm{pRFI}\right]= \\
& -1\left[\mathrm{DMI}_{\text {adj }}-b_{1}(\mathrm{MilkE}-\overline{\mathrm{MilkE}})\right] .
\end{aligned}
$$

Again the term $b_{2}(\mathrm{MBW}-\overline{\mathrm{MBW}})$ reflects the energy required for maintenance. The "-1" multiplier in Equation [17] renders a "higher is better" measure that may be more attractive to industry than the "lower is better" that characterizes pRFI or gRFI, for example.

By analogy, one could readily come up with genetic feed saved (GFS) alternative to pFS starting from Equation [10c] and as defined below in Equation [18].

$$
u_{g F S}=-1\left(b_{2}^{(u)} u_{M B W}+u_{g R F I}\right)=-1\left(u_{D M I}-b_{1}^{(u)} u_{M i l k E}\right) .
$$

For reasons that we have already presented in favor of gRFI over pRFI, we might focus primarily on gFS instead of pFS. The corresponding contrast vector used to derive the genetic merit for gFS using Equation [18] is $\mathbf{k}_{g F S(u)}^{\prime}=\left[\begin{array}{ccc}\hat{b}_{1}^{(u)} & 0 & -1\end{array}\right]$ based on the same multiple trait system and same 3 component FE traits used to derive pRFI and gRFI. Similarly, the corresponding contrast vector for $\mathrm{pFS}$ is $\mathbf{k}_{p F S}^{\prime}=\left[\begin{array}{lll}\hat{b}_{1} & 0 & -1\end{array}\right]$. Using similar algebra to that previously shown for inferring the genetic correlation between gRFI and pRFI, the estimated genetic correlation between $\mathrm{gFS}$ and $\mathrm{pFS}$ based on the provided $\hat{\mathbf{G}}$ in Equation [4] is $\operatorname{corr}\left(\mathbf{k}_{g F S(u)}^{\prime} \mathbf{u}_{c o m p}, \mathbf{k}_{p F S}^{\prime} \mathbf{u}_{\text {comp }}\right)=0.99$, suggesting that EBV rankings of animals would differ very little between gFS and pFS.

\section{Residual Milk Energy}

Coleman et al. (2010) proposed a FE measure that they referred to as residual milk solids or residual milk energy (RME). They derived RME in a manner very similar to that of pRFI except the roles of the 2 traits DMI and MilkE are reversed; that is, MilkE is adjusted for DMI, with corresponding partial regression coefficient $c_{1}$, and adjusted for MBW, with partial regression corresponding coefficient $c_{2}$, to derive RME as the estimated residual. Just as with RFI and FS, one could alternatively derive a phenotypic RME (i.e., pRME) as in Coleman et al. (2010) or a genetic RME 
(i.e., gRME). For example, consider a modification of Equation [10c] to derive gRME, with genetic merit $u_{g R M E}$, rather than gRFI as in Equation [19].

$$
\begin{aligned}
& u_{\text {MilkE }}=c_{1}^{(u)} u_{D M I}+c_{2}^{(u)} u_{M B W}+u_{g R M E} ; \\
& e_{\text {MilkE }}=c_{1}^{(e)} e_{D M I}+c_{2}^{(e)} e_{M B W}+e_{g R M E} .
\end{aligned}
$$

Of course, Equation [19] is based on the recursive relationships defined from DMI to MBW to MilkE rather than from MilkE to MBW to DMI as defined in Equations [10a], [10b], and [10c].

The estimates of the coefficients in Equation [19] used to determine $u_{g R M E}$ and $e_{g R M E}$ can be based on partitioning $\hat{\mathbf{G}}$ and $\hat{\mathbf{R}}$, as was done similarly for gRFI earlier in Equations [11] and [12]. This process would lead to the corresponding estimates of $\quad \hat{c}_{1}^{(u)}=1.32 \mathrm{Mcal} / \mathrm{kg}, \quad \hat{c}_{2}^{(u)}=-0.13 \mathrm{Mcal} / \mathrm{kg}^{0.75}$, $\hat{c}_{1}^{(e)}=0.94 \mathrm{Mcal} / \mathrm{kg}$, and $\hat{c}_{2}^{(e)}=0.01 \mathrm{Mcal} / \mathrm{kg}^{0.75}$. The determination of pRME would be similarly based on partitioning $\hat{\mathbf{P}}$ analogous to that done in Equation [7] to estimate $c_{1}$ and $c_{2}$. The corresponding estimates are $\hat{c}_{1}$ $=1.04 \mathrm{Mcal} / \mathrm{kg}$ and $\hat{c}_{2}=-0.04 \mathrm{Mcal} / \mathrm{kg}^{0.75}$. Similar to what was observed for the gRFI versus pRFI coefficients, note that $\hat{c}_{1}$ lies between $\hat{c}_{1}^{(u)}$ and $\hat{c}_{1}^{(e)}$, whereas $\hat{c}_{2}$ lies between $\hat{c}_{2}^{(u)}$ and $\hat{c}_{2}^{(e)}$.

Now the contrast vector linking the genetic merit of gRME to the 3 key FE component traits is $\mathbf{k}_{g R M E(u)}^{\prime}=\left[\begin{array}{ccc}1 & -\hat{c}_{2}^{(u)} & -\hat{c}_{1}^{(u)}\end{array}\right]$, whereas the corresponding contrast vector linking pRME to the 3 key component traits is $\mathbf{k}_{p R M E}^{\prime}=\left[\begin{array}{lll}1 & -\hat{c}_{2} & -\hat{c}_{1}\end{array}\right]$. The corresponding estimated genetic correlation

$$
\operatorname{corr}\left(\mathbf{k}_{g R M E(u)}^{\prime} \quad \mathbf{u}_{c o m p, i}, \quad \mathbf{k}_{p R M E}^{\prime} \quad \mathbf{u}_{c o m p, i}\right)
$$

between pRME and gRME is 0.95, which is somewhat lower than that between pRFI and gRFI or between pFS and gFS as we reported earlier. This lower estimate may be, in part, due to the greater discrepancy between the genetic and residual correlations between MilkE and MBW as we noted earlier or, equivalently, the relatively large discrepancy between $\hat{c}_{2}^{(u)}$ and $\hat{c}_{2}^{(e)}$.

\section{Gross Feed Efficiency}

As illustrated earlier, a classical measure of $\mathrm{FE}$ is GFE or other similar ratios of product over input or input over product. However, given that income over feed costs (IOFC) is far more likely to be linearly related to the key FE component traits of DMI, MilkE, and, perhaps less so, MBW, then IOFC is more likely to be related to any other linear functions of these key component traits such as FS, RFI, or RME. Furthermore, as noted by several researchers (Berry and Crowley, 2013; VandeHaar et al., 2016), ratio measures can be misleading as they generally fail to take into account BW changes. Additionally, direct selection on ratio traits can lead to unintended consequences on the numerator or denominator traits specified on that ratio (Gunsett, 1984). At any rate, Zetouni et al. (2017) have demonstrated that effective multiple trait selection for FE component traits, as we advocate here, should also realize the greatest genetic response for ratio traits such as GFE.

Recently, Shirali et al. (2018) have demonstrated how inferences from a multi-trait genetic evaluation model on FE component traits can be used to provide genetic evaluations for ratio traits such as GFE. Given that GFE is a nonlinear (i.e., ratio) function of $\mathbf{u}_{\text {comp }}$, contrast vectors cannot be used to derive EBV nor their associated reliabilities using a standard multiple trait BLUP system. Shirali et al. (2018) thereby proposed a very effective, albeit computationally expensive, Bayesian strategy to contend with that particular issue.

\section{Deriving Genetic Correlations of Feed Efficiency Traits}

Let $\mathbf{K}_{u}^{\prime}=\left[\begin{array}{llll}\mathbf{I}_{3} & \mathbf{k}_{g R F I(u)} & \mathbf{k}_{g F S(u)} & \mathbf{k}_{g R M E(u)}\end{array}\right]$ such that then

$$
\mathbf{K}_{u} \mathbf{u}_{\text {comp }}=\left[\begin{array}{llllll}
u_{M i l k E} & u_{M B W} & u_{D M I} & u_{g R F I} & u_{g F S} & u_{g R M E}
\end{array}\right]^{\prime}
$$

jointly represents the genetic merit on MilkE, MBW, DMI, gRFI, gFS, and gRME. Then the genetic variance covariance matrix of those effects as a function of the 3 key component $\mathrm{FE}$ traits can be written as $\mathbf{K}_{u} \mathbf{G K}_{u}^{\prime}$, which we can estimate using $\mathbf{K}_{u} \widehat{\mathbf{G}} \mathbf{K}_{u}^{\prime}$. This, in turn, can be readily demonstrated to lead to an estimated correlation matrix $\hat{\rho}\left[\mathbf{K}_{u} \mathbf{u}_{\text {comp }}\right]$ as in Equation [20].

$$
\begin{gathered}
\hat{\rho}\left[\mathbf{K}_{u} \mathbf{u}_{\text {comp }}\right]=\hat{\rho}\left[\begin{array}{c}
u_{\text {MilkE }} \\
u_{M B W} \\
u_{D M I} \\
u_{g R F I} \\
u_{g F S} \\
u_{g R M E}
\end{array}\right]= \\
{\left[\begin{array}{cccccc}
1 & 0.02 & 0.64 & 0 & -0.01 & 0.68 \\
0.02 & 1 & 0.48 & 0 & -0.63 & 0 \\
0.64 & 0.48 & 1 & 0.60 & -0.77 & 0 \\
0 & 0 & 0.60 & 1 & -0.78 & -0.73 \\
-0.01 & -0.63 & -0.77 & -0.78 & 1 & 0.57 \\
0.68 & 0 & 0 & -0.73 & 0.57 & 1
\end{array}\right] .}
\end{gathered}
$$


Note, for example, that the genetic correlation between gRME with each of MBW and DMI in $\hat{\rho}\left[\mathbf{K}_{u} \mathbf{u}_{\text {comp }}\right]$ is zero, precisely because of the genotypic regressions that also lead to a zero genetic correlation between gRFI with MilkE and MBW as previously noted. It is interesting to note the strong genetic correlations between gFS with MBW, DMI, gRFI, and gRME. In other words, selecting directly on a higher gFS should lead to a lower MBW and DMI as well as a lower (i.e., more favorable) gRFI, higher gRME, and little effect on MilkE. Conversely, selecting directly for lower gRFI should lead to favorable changes in DMI, gFS, and gRME with no effects on MilkE and MBW.

One could determine the corresponding residual variance-covariance matrices using $\mathbf{K}_{e}^{\prime}=\left[\begin{array}{llll}\mathbf{I}_{3} & \mathbf{k}_{g R F I(e)} & \mathbf{k}_{g F S(e)} & \mathbf{k}_{g R M E(e)}\end{array}\right]$. The corresponding estimated heritabilities for DMI and the corresponding derivative FE traits can be derived from the corresponding diagonals

$\operatorname{diag}\left(\mathbf{K}_{u} \hat{\mathbf{G}} \mathbf{K}_{u}^{\prime}\right) /\left[\operatorname{diag}\left(\mathbf{K}_{u} \hat{\mathbf{G}} \mathbf{K}_{u}^{\prime}\right)+\operatorname{diag}\left(\mathbf{K}_{e} \hat{\mathbf{R}} \mathbf{K}_{e}^{\prime}\right)\right] . \quad$ Ordered from highest to lowest estimates, the corresponding heritabilities for the various $\mathrm{FE}$ traits would then be $\hat{h}_{D M I}^{2}=0.23, \hat{h}_{g F S}^{2}=0.21, \hat{h}_{g R M E}^{2}=0.17$, and $\hat{h}_{g R F I}^{2}=$ 0.15. It is important to quickly add here that one can readily create extensions of $\mathbf{K}_{u}$ and $\mathbf{K}_{e}$ to determine DIM-specific genetic and residual correlations between these same traits based on multiple trait random regression models that specify the genetic effects of each of the 3 key component traits, MilkE, MBW, and DMI, as random polynomial effects on DIM (Hurley et al., 2017).

\section{MULTIPLE TRAIT RELIABILITIES}

By definition, the accuracy of an EBV is its correlation with the corresponding true breeding value, whereas reliability is defined to be the square of the accuracy. Genetic evaluation reliabilities for a single trait genetic evaluation are typically derived from the prediction error variance (PEV) as determined from the mixed model equations. By extension, prediction error (co) variances from multiple trait mixed model equations can be similarly used to determine reliabilities not only of the key component FE traits, MilkE, MBW, and DMI, defined within the mixed model system but also any derivative FE traits such as gRFI, gFS, or gRME, for example, that are linear functions of the component traits.

Although most current genetic evaluations, and thereby reliabilities, are based on the use of genomic information, we focus simply on relative assessments of reliabilities between the various traits using pedigree- based assessments such as the number of half sib progeny having records. Relative comparisons between the various $\mathrm{FE}$ traits for genomic-based reliabilities should not be vastly different from pedigree-based reliabilities. For example, it is well recognized that the reliability of a sire EBV is dependent upon the heritability $\left(h^{2}\right)$ of the trait and the number $(n)$ of half sib progeny (Cameron, 1997) as illustrated for a non-inbred sire in Equation [21].

$$
\text { Reliability }(\mathrm{EBV})=0.5 \frac{\frac{1}{2} n h^{2}}{1+(n-1) \frac{1}{4} h^{2}} .
$$

More formally, this could be derived from the mixed model equations as in Equation [22]:

$$
\text { Reliability }(\mathrm{EBV})=\frac{\sigma_{u}^{2}-\mathrm{PEV}(\mathrm{EBV})}{\sigma_{u}^{2}} .
$$

Here $\mathrm{PEV}(\mathrm{EBV})$ is based on the inverse of the mixed model equations coefficient matrix pertaining to the diagonal element or animal (i.e., EBV) of interest. For the reliability of the corresponding EBV, EBV = $\left[\begin{array}{lll}\mathrm{EBV}_{1} & \mathrm{EBV}_{2} & \mathrm{EBV}_{3}\end{array}\right]^{\prime}$, of 3 traits, say the key component traits defining FE $(1=$ MilkE, $2=\mathrm{MBW}$, and 3 $=\mathrm{DMI})$, within a multiple trait genetic evaluation system, the appropriate extension would be as in Equation [23].

$$
\operatorname{Reliability}(\mathbf{E B V})=\frac{\operatorname{diag}[\mathbf{G}-P E V(\mathbf{E B V})]}{\operatorname{diag}(\mathbf{G})} .
$$

Here $\operatorname{PEV}(\mathbf{E B V})$ is based on the $3 \times 3$ component of the inverse of the mixed model equations coefficient matrix pertaining to the block diagonal for the 3 key component traits on the animal of interest and $\mathbf{G}$ was defined in Equation [3]. The reliability for a derivative trait such as $\mathbf{k}^{\prime} \mathbf{u}_{\text {comp }}$, where $\mathbf{k}^{\prime}$ could pertain either to $\mathbf{k}_{p R F I}^{\prime}, \mathbf{k}_{g R F I(u)}^{\prime}, \mathbf{k}_{p F S}^{\prime}, \mathbf{k}_{g F S}^{\prime}, \mathbf{k}_{p R M E}^{\prime}$, or $\mathbf{k}_{g R M E}^{\prime}$, can be determined as Reliability $\left(\mathbf{k}^{\prime} \mathbf{u}_{\text {comp }}\right)=\mathbf{k}^{\prime}$ Reliability $(\mathbf{E B V}) \mathbf{k}$ using Equation [23]. Again, Equations [21], [22], and [23] pertain to non-inbred sires with only minor modifications required for inbred animals.

It seems instructive to characterize sire reliabilities of the various $\mathrm{FE}$ traits as a function of the number of half sib daughters. We assume here that $\mathbf{G}=\hat{\mathbf{G}}$ and $\mathbf{R}$ $=\hat{\mathbf{R}}$ as provided in Equation [4]. Refer to Figure 1, which has 3 panels. In all 3 panels, every daughter has records on both MBW and MilkE. For the situation 
where records are available for all 3 key component traits on all progeny (panel a), both DMI and gFS have nearly equal reliabilities that are both substantially larger than those for gRFI and gRME, which are, in turn, nearly equal to each other. When only $50 \%$ of the daughters have DMI records (panel b), there is more separation in reliabilities between the 4 traits with DMI still on top, followed by gFS, gRME, and then gRFI. When none of the daughters have DMI records (panel c), reliabilities for all traits fall substantially as expected. Of particular notice, the reliability of genetic evaluations for gRFI is equal to zero for any number of daughters having records on MilkE and MBW, whereas DMI dominates all of the other traits for reliabilities. This should be not too surprising looking at the genetic correlation matrix provided in Equation [20]; that is, genetic merit for DMI should be best predicted in all 3 scenarios because of its higher heritability and its high genetic correlations with MilkE and MBW. Conversely, gRFI has zero genetic correlation with MilkE and MBW and hence its EBV reliability more critically depends on good recording of DMI phenotypes. A similar observation would be drawn for pRFI as for gRFI given the large genetic correlation between these 2 specifications as previously noted.

\section{SELECTION INDEXES}

\section{Economic Weight Determinations on Feed Efficiency Traits}

A question that invariably comes up is which FE trait to include within a selection index. As Kennedy et al. (1993) indicated earlier, properly specified selection indexes based on either DMI, pRFI, or gRFI should be identical in terms of total net merit prediction and reliability. Some concerns have been expressed that selection for RFI could lead to undesired trends in other economically important traits (Hurley et al., 2017). However, those concerns should be readily addressed if all economically important traits are incorporated within a selection index with "known" genetic covariances specified between all traits, a point already well borne out by Kennedy et al. (1993) and later by Lu et al. (2015).

For simplicity of presentation, suppose a particular selection index, say, an efficiency index (EI) closely related to IOFC, only involves the key component traits MilkE, MBW, and DMI. Then

$$
\mathrm{EI}=v_{1} \mathrm{EBV}_{\text {MilkE }}+v_{2} \mathrm{EBV}_{M B W}+v_{3} \mathrm{EBV}_{D M I} .
$$

Here $v_{1}, v_{2}$, and $v_{3}$ are economic weights that should be closely tied to commodity prices, whereas $\mathrm{EBV}_{\text {MilkE }}$,
$\mathrm{EBV}_{M B W}$, and $\mathrm{EBV}_{D M I}$ are corresponding EBV for the 3 traits. Outside of fluctuating milk and feed prices, and variable nutrient contents of feedstuffs across regions and herds, it should be reasonably straightforward to determine $v_{1}$ and $v_{3}$. It is perhaps more challenging to specify $v_{2}$ given that this would need to reflect, for example, the revenue (e.g., extra beef income) balanced against the costs (e.g., greater housing costs) associated with greater body size. Nevertheless, $v_{2}$ should not include feed costs since they are already factored into $v_{3}$; indeed, this sort of double counting needs to be avoided in selection indexes as previously warned by Veerkamp (1998). The same would be true, for example, involving any health costs associated with larger or smaller BW if that particular component of health was already included in the index. Ideally, $v_{1}, v_{2}$, and $v_{3}$ would be expressed on a lifetime scale basis as per the Net Merit Index (Van Raden et al., 2018a), but for simplicity of our exposition as we indicated earlier, we keep the index on the same scale (profit/d within 50-200 DIM) as the traits inferred by Lu et al. (2018) to allow direct incorporation of $\hat{\mathbf{G}}$ from Equation [4].

Assume, for simple illustration, that $v_{1}=\$ 0.60 / \mathrm{Mcal}$, $v_{2}=0$, and $v_{3}=-\$ 0.25 / \mathrm{kg}$. The value for $v_{1}$ is based on milk fat prices of $\$ 5.50 / \mathrm{kg}$ and $9.29 \mathrm{Mcal} / \mathrm{kg}$ fat and roughly corresponds to $\$ 19 / \mathrm{cwt}$ for $3.5 \%$ fat and $3.0 \%$ protein-corrected milk. Realizing that MilkE is also a function of protein and lactose, albeit with lower combustible energies per kilogram relative to fat, we make the simplifying assumption here that all MilkE is fat. Indeed, future studies on the genetics of dairy cattle FE should entail a multiple trait system that separately includes fat, protein, and lactose, instead of a composite MilkE, as key component traits of FE, each having their own phenotypic, genetic, or residual partial regression coefficients involving DMI, thereby expanding the dimension of $\mathbf{G}$ and $\mathbf{R}$ and, hence, Equations [7], [11], and [12], for example.

Suppose that the EBV for a particular cow on the 3 key component FE traits are $+1.6 \mathrm{Mcal} / \mathrm{d}$ for MilkE, $+5.0 \mathrm{~kg}^{0.75}$ for MBW, and $+1.0 \mathrm{~kg} / \mathrm{d}$ for DMI. Then that cow's EI is EI $=0.60(+1.6)+0(+5.0)-0.25(+1.0)=$ $+\$ 0.71 / \mathrm{d}$. Consider the same EI now but written as a function of gRFI using Equations [10c] and [24] and as derived in Equation [25].

$$
\begin{aligned}
& \mathrm{EI}=v_{1} \mathrm{EBV}_{M i l k E}+v_{2} \mathrm{EBV}_{M B W}+v_{3}\left(\hat{b}_{1}^{(u)} \mathrm{EBV}_{M i l k E}+\hat{b}_{2}^{(u)} \mathrm{EBV}_{M B W}+\mathrm{EBV}_{g R F I}\right) \\
& =\left(v_{1}+v_{3} \hat{b}_{1}^{(u)}\right) \mathrm{EBV}_{M i l k E}+\left(v_{2}+v_{3} \hat{b}_{2}^{(u)}\right) \mathrm{EBV}_{M B W}+v_{3} \mathrm{EBV}_{g R F I} \\
& =[0.60-0.25(0.40)] \mathrm{EBV}_{M i l k E}+[0-0.25(0.10)] \mathrm{EBV}_{M B W}+(-0.25) \mathrm{EBV}_{g R F I} \\
& =0.50 \mathrm{EBV}_{M i l k E}-0.025 \mathrm{EBV}_{M B W}-0.25 \mathrm{EBV}_{g R F I} .
\end{aligned}
$$


Now note that the EBV for gRFI on this cow borrowing from Equation [10c] is $\mathrm{EBV}_{g R F I}=\mathrm{EBV}_{D M I}-$ $\hat{b}_{1}^{(u)} \mathrm{EBV}_{\text {MilkE }}-\hat{b}_{2}^{(u)} \mathrm{EBV}_{M B W}=+1.0-0.40(+1.6)-$ $0.10(+5.0)=-0.14$. Hence, as also previously demonstrated by $\mathrm{Lu}$ et al. (2015), this particular cow's EI, written now as a function of its EBV for gRFI, is unchanged with $\mathrm{EI}=0.5(1.6)-0.025(5.0)-0.25(-0.14)$ $=+\$ 0.71 / \mathrm{d}$. Absolute and relative (Van Raden et al., 2018a) economic weights on each of MilkE, MBW, and the corresponding FE trait (i.e., DMI, gRFI, gFS, or gRME) specified in the index are provided in Table 1. It is interesting to note from Table 1 that, whereas the absolute economic weights (i.e., $\$ 0.25 / \mathrm{kg}$ ) should not differ between DMI, gRFI, and gFS, their relative economic weights do change depending on which of these FE traits is included in the index, even though the EI for any particular cow is unchanged in all 3 cases. Furthermore, whereas $v_{2}=0$ implies zero economic weight on MBW when DMI or gFS is included in the index, a nonzero economic weight is placed on MBW when gRFI is alternatively included in the index as the economic cost for maintenance requirement is then placed on MBW.

The current Net Merit Index uses BW composite, which is considered to be a linear function of BW (Van Raden et al., 2018b) rather than of MBW. Given that BW is nearly a linear function of MBW within the normal range of Holstein cow BW (Figure 2), it seems prudent to conduct future dairy cattle FE genetics analyses modeling BW rather than MBW as an energy sink. This would better facilitate the incorporation of the multiple trait analysis strategies presented in this paper for deriving various FE traits like gFS or gRFI from economically important FE component traits such as fat, protein, and BW (or BW composite), which are already specified within the Net Merit Index.
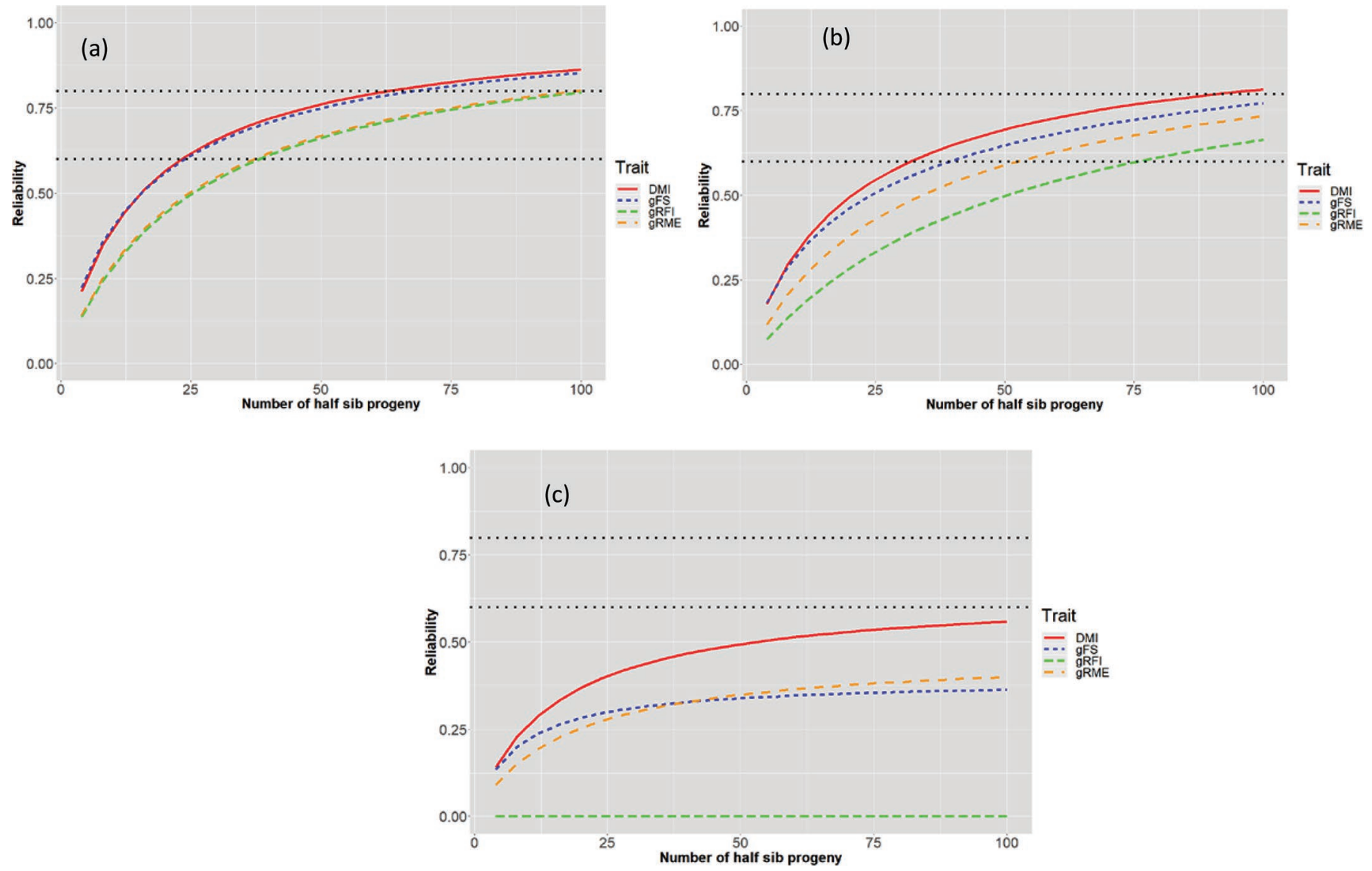

Figure 1. Reliabilities of sire genetic evaluations for DMI, genetic feed saved (gFS), genetic residual feed intake (gRFI), and genetic residual milk energy (gRME) as a function of number of half sib daughters and based on estimated genetic and residual (co)variances between DMI, milk energy (MilkE), and metabolic body weights (MBW) from Lu et al. (2018). All daughters have MilkE and MBW records with (a) having $100 \%$ of daughters with DMI records, (b) having $50 \%$ of daughters with DMI records, and (c) having no daughters with DMI records. Horizontal reference lines provided at reliabilities of 0.60 and 0.80 . 


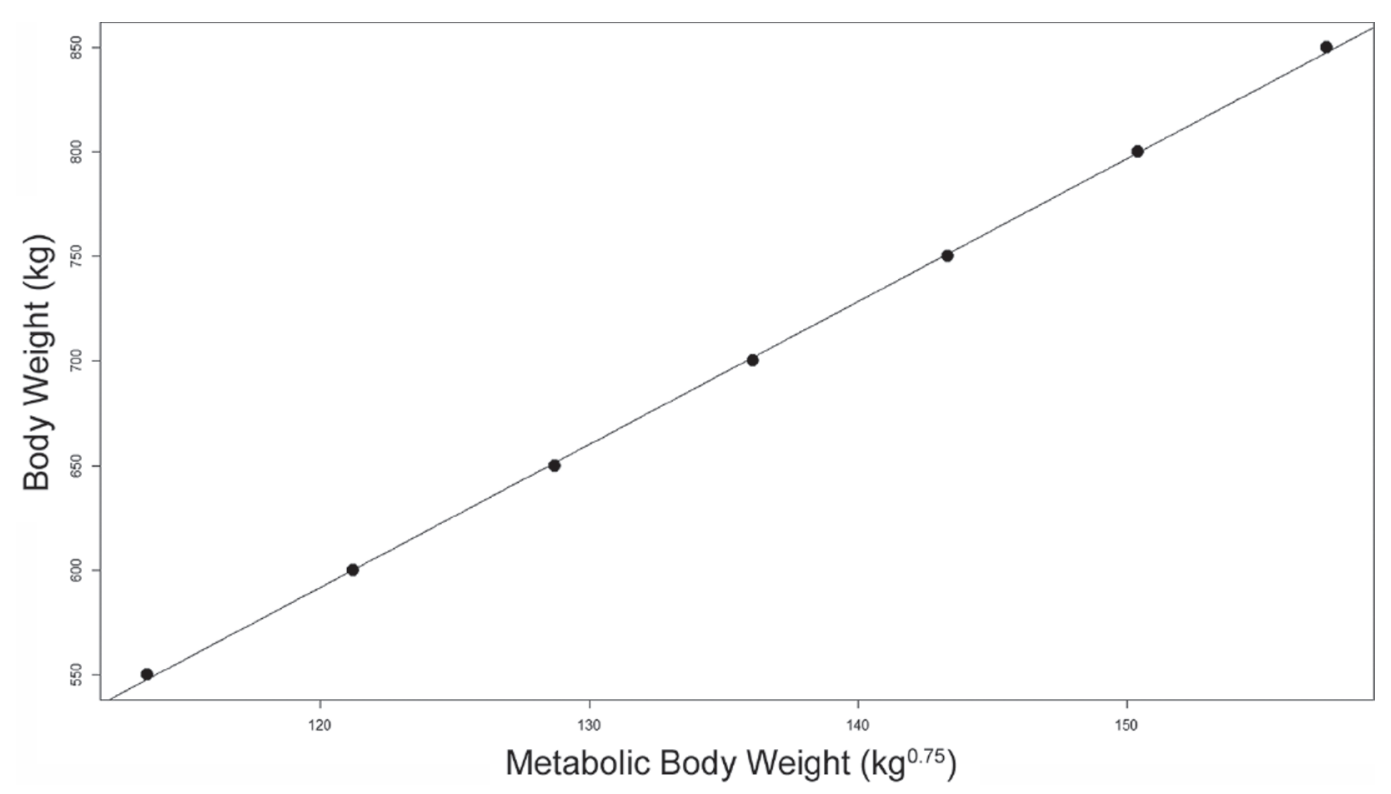

Figure 2. Plot of BW $(\mathrm{kg})$ versus metabolic BW $\left(\mathrm{kg}^{0.75}\right)$ over a normal range of Holstein cow weights $(550-850 \mathrm{~kg})$. Least squares line of best fit has slope $=6.84 \mathrm{~kg} / \mathrm{kg}^{0.75}$.

\section{Correlated Response to Selection}

The expected change in EI in one sire generation of selection can be determined as $\Delta \mathrm{EI}=\sqrt{\text { reliability }} \times i \times \sigma_{I}$, where reliability could pertain to the average sire reliability of the index, $\sigma_{I}=\sqrt{\mathbf{v}^{\prime} \mathbf{G v}}$ is the standard deviation of the index for $\mathbf{v}=\left[\begin{array}{lll}v_{1} & v_{2} & v_{3}\end{array}\right]^{\prime}$ being the vector of economic weights on the key component FE traits, and $i$ is the selection intensity (Falconer and Mackay, 1996). For the top $5 \%$ of sires selected, the selection intensity would be $i=2.06$. Suppose the average reliability of the sire index (EI) is 0.60 . Then the expected response in the index per sire generation is $\Delta \mathrm{EI}=\sqrt{\text { reliability }} \times i \times \hat{\sigma}_{I}=\$ 1.34 /$ gen, where $\hat{\sigma}_{I}=\sqrt{\mathbf{v}^{\prime} \hat{\mathbf{G}} \mathbf{v}}=\sqrt{0.706}$ using the specifications provided earlier. Furthermore, the correlated response to index selection on each of the traits can be determined as $\mathbf{K}_{u}^{\prime} \mathbf{G v} \sqrt{\text { reliability }} i / \sqrt{\mathbf{v}^{\prime} \mathbf{G v}}$, extending results in Kennedy et al. (1993). Using the provided specifications including $\mathbf{G}=\hat{\mathbf{G}}$ and a selection intensity of $5 \%(i=$ 2.06), the correlated responses in MilkE, MBW, DMI, gRFI, gFS, and gRME are then calculated to be 2.54 Mcal $/ \mathrm{d},-1.08 \mathrm{~kg}^{0.75}, 0.74 \mathrm{~kg} / \mathrm{d},-0.18 \mathrm{~kg} / \mathrm{d}, 0.29 \mathrm{~kg} / \mathrm{d}$, and $1.43 \mathrm{Mcal} / \mathrm{d}$, respectively, in one sire generation. Hence, breeding programs based on reasonably specified selection indexes would not necessarily decrease DMI over time; however, BW should decline while genetically improving the various measures of $\mathrm{FE}$ (i.e., gRFI, gFS, or gRME).

A summary of the various "genetic regression" FE traits (gRFI, gFS, and gRME), their relationships to the component traits, and the effect of single trait selection on the $\mathrm{FE}$ traits with respect to correlated changes in the component traits and the EI index are provided in Table 2 . Note that direct selection for lower DMI leads to correlated declines in MilkE and MBW and the index. As anticipated, direct selection for gRFI decreases DMI without any change in MilkE and MBW

Table 1. Selection index economic weights on milk energy (MilkE), metabolic body weight (MBW), and a feed efficiency (FE) trait being DMI, genetic residual feed intake (gRFI), genetic feed saved (gFS), or genetic residual milk energy (gRME) ${ }^{1}$

\begin{tabular}{lccc}
\hline $\begin{array}{l}\text { FE trait included } \\
\text { in index }\end{array}$ & $\begin{array}{c}\text { Actual (relative) economic weight } \\
\text { placed on MilkE in } \$ / \text { Mcal }\end{array}$ & $\begin{array}{c}\text { Actual (relative) economic weight } \\
\text { placed on MBW in } \$ / \mathrm{kg}^{0.75}\end{array}$ & $\begin{array}{c}\text { Actual (relative) economic weight } \\
\text { placed on FE trait }\end{array}$ \\
\hline DMI & $+0.60(79)$ & $0(0)$ & $-0.25(21)$ \\
gRFI & $+0.50(75)$ & $-0.024(11)$ & $-0.25(14)$ \\
gFS & $+0.50(80)$ & $-0.024(12)$ & $+0.25(20)$ \\
gRME & $+0.41(67)$ & $+0.19(21)$ & +19 \\
\hline
\end{tabular}

${ }^{1}$ Economic weights on MilkE, MBW, and DMI were assumed to be $\$ 0.60 /$ Mcal, 0 , and $-\$ 0.25 / \mathrm{kg}$, respectively, as per the first row in the table. Genetic and residual (co)variances between MilkE, MBW, and DMI were assumed to be those estimated by Lu et al. (2018). Relative weights (\%) computed as per Van Raden et al. (2018a) are provided in parentheses. 
Table 2. Implications of single trait selection on various feed efficiency (FE) traits, being DMI, genetic residual feed intake (gRFI), genetic feed saved (gFS), or genetic residual milk energy (gRME) for genetic change in one sire generation on milk energy (MilkE), metabolic body weight $(\mathrm{MBW}), \mathrm{DMI}$, and selection index as a function of MilkE, MBW, and DMI with economic weights $\$ 0.60 / \mathrm{Mcal}, 0$, and $-\$ 0.25 / \mathrm{kg}, \mathrm{respectively}{ }^{1}$

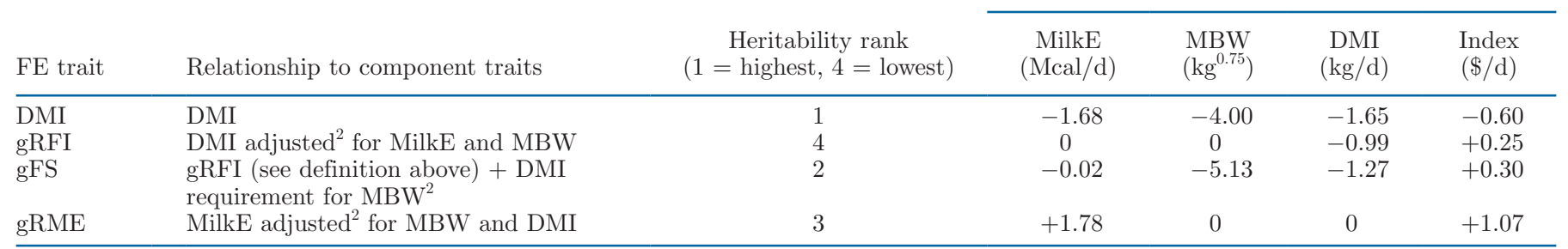

${ }^{1}$ Relationships of FE to component traits and their most probable relative ranking on heritabilities are also provided. Genetic (co)variances between MilkE, MBW, and DMI assumed to be those estimated by Lu et al. (2018). Selection intensity is based on top $5 \%$ in favorable direction for $\mathrm{FE}$ trait and reliabilities of genetic evaluations for selection on corresponding $\mathrm{FE}$ trait $=60 \%$.

${ }^{2}$ Adjustments based on genetic regressions.

and only a moderate positive change in the index. Direct selection for gFS decreases both MBW and DMI with a near-zero change in MilkE and a moderate positive change in the index. Interestingly, direct selection for gRME leads to the most favorable change in the index due to the strong correlated response in MilkE with no change in MBW or DMI; nevertheless, direct selection on gRME would still be suboptimal to selection on an index with properly specified economic weights. It can be readily derived from the provided $\widehat{\mathbf{G}}$ that the estimated genetic correlations between DMI, gRFI, gFS, and gRME with the EI index are +0.39 , $-0.23,+0.28$, and +0.82 , respectively, thereby agreeing with the general observations on correlated genetic changes drawn from Table 2. Note that because DMI has a strong positive genetic correlation with MilkE, it is also positively correlated with the index even though the economic weight on DMI is negative.

\section{CHALLENGES}

\section{Heterogeneity of Effects}

All 3 key derivative FE traits, RFI, FS, or RME, are based on partial regression inferences, whether at the phenotypic level (i.e., pRFI, pFS, or pRME) or at the genotypic level (i.e., gRFI, gFS, or gRME). The standard use of OLS or the conditional normal theory method presented by Kennedy et al. (1993) to derive these partial regression coefficients generally presume a constant relationship across environments for example (Veerkamp et al., 1995; Berry and Crowley, 2013). However, given that DMI is typically recorded in kilograms, that rations can vary substantially in their nutrient composition by DM weight, that herds can differ substantially in their management and environment, and that even energy partitioning of DMI into MilkE, for example, can depend upon stage of lactation, it is a rather tenuous assumption that these partial regression coefficients are homogeneous.

Tempelman et al. (2015), for example, inferred heterogeneous coefficients for $b_{1}$ ranging from 0.29 to $0.47 \mathrm{~kg} / \mathrm{Mal}$ and for $b_{2}$ ranging from 0.06 to $0.16 \mathrm{~kg} /$ $\mathrm{kg}^{0.75}$ across research herds from 4 different countries. Additionally, they determined that variation in ration specific slopes for $b_{1}$ and $b_{2}$ was statistically significant, suggesting a range as wide as $0.30 \mathrm{~kg} /$ Mcal between different rations for $b_{1}$ even within research stations.

$\mathrm{Lu}$ et al. (2017) took this a step further for gRFI, inferring heterogeneity in $b_{1}^{(u)}, b_{1}^{(e)}, b_{2}^{(u)}$, and $b_{2}^{(e)}$, determining that there was substantial heterogeneity in $b_{2}^{(e)}$ across stations ranging from -0.04 to $0.18 \mathrm{~kg} / \mathrm{kg}^{0.75}$. Lu et al. (2017) also discovered significant and substantial heteroskedasticity in $\sigma_{u_{g R F I}}^{2}$ and $\sigma_{e_{g R F I}}^{2}$ across stations ranging from 0.47 to $1.28 \mathrm{~kg}^{2}$ for $\sigma_{u_{g R F I}}^{2}$ and from 0.85 to $3.01 \mathrm{~kg}^{2}$ for $\sigma_{e_{q R F I}}^{2}$. Additionally, $\sigma_{e_{q R F I}}^{2}$ was inferred to be nearly 3 times larger for multiparous cows as opposed to primiparous cows leading to a 2 -fold difference in $h_{q R F I}^{2}$ between the 2 parities. Lu et al. (2017) estimated the coefficient of variation for ration-specific $\sigma_{u_{q R F I}}^{2}$ within stations to be $0.37 \pm 0.08$. In other words, relative to an average value $\bar{\sigma}_{u_{g R F I}}^{2}$ for $\sigma_{u_{g R F I}}^{2}$, the standard deviation of these ration-specific genetic variances were estimated to be $37 \%$ of the magnitude of $\bar{\sigma}_{u_{g R F I}}^{2}$ within station. Multifactorial assessments of heterogeneous $\sigma_{e_{g R F I}}^{2}$ could further allow investigation into factors (e.g., feed recording systems) responsible for poor measurement error, for example. Lu et al. (2017) also discovered that modeling heterogeneous genetic/residual partial 
regressions and variance components for gRFI potentially uncovers proportionately greater genetic variability such that the average heritability estimate for gRFI across research stations in their study was 0.44. This average estimate is more than double the average estimated heritability reported for RFI in the literature.

Deriving pRFI or gRFI implies a certain defined relationship between DMI and the energy sink traits (MilkE and MBW), specifically that these relationships are linear (Berry and Crowley, 2013). Indeed, if the partial genetic and residual relationships are not linear between these traits, then the use of multiple trait analyses and selection indexes are completely invalidated as these methods are entirely predicated on relationships between traits being linear. However, we, as others, have not detected any evidence of such nonlinearity, provided that random regression specifications are used to model, for example, ration-specific partial regressions of DMI on MilkE and MBW (Tempelman et al., 2015; Lu et al., 2017). That is, the use of random regression specifications for ration- or herd-specific heterogeneity on partial regressions mitigates the appearance of nonlinearity that would otherwise exist if this heterogeneity is ignored. Investigators should carefully monitor and report estimates of the corresponding partial regression coefficients in any quantitative genetic studies involving RFI or FS, for example. For example, Davis et al. (2014) derived RFI separately for each of 4 consecutive trials of New Zealand Holstein Friesian cattle, noting substantial heterogeneity in these partial regression coefficients, even though cows were all presumably fed the same diets across these trials. Sometimes inferred partial regression relationships can seem non-intuitive; for example, Davis et al. (2014) inferred a significant negative relationship between DMI and $\Delta \mathrm{BW}$ within one trial.

The coefficients $b_{1}$ and $b_{2}$ and their gRFI counterparts $b_{1}^{(u)}, b_{1}^{(e)}, b_{2}^{(u)}$, and $b_{2}^{(e)}$ may be systematically changing over time. For example, a recent study by de Souza et al. (2019), using a much larger data set than Lu et al. (2018) and weekly records rather than 28-d records, suggests that $b_{2}$ may be substantially larger than the $0.0968 \mathrm{~kg} / \mathrm{kg}^{0.75}$ determined by NRC (2001) and the $b_{2}$ estimate reported by $\mathrm{Lu}$ et al. (2018). VandeHaar and Tempelman (2016), citing Moraes et al. (2015), observed that $b_{2}$ may have increased in recent decades, suggesting that there may be a positive correlation between $b_{2}$ and selection for milk yield. These changes imply that selection indexes like the Net Merit Index will need to adaptively change not only with changes in economic values and the addition of new traits but also with emerging trends in phenotypic or genetic relationships between component traits in the index. These trends and the noted heterogeneities in the partial regression coefficients across herds and rations should also provide some caution to researchers who determine derivative traits that are based on constant and "known" coefficients reported elsewhere. For example, such assumptions are typically invoked in genetic studies involving energy balance that specify maintenance costs as a known constant linear function of MBW (Harder et al., 2019; Krattenmacher et al., 2019) or even definitions of RFI based on using feed tables (Berry and Crowley, 2013) instead of using estimated partial regression relationships as we would strongly advise.

Heterogeneity in $b_{1}^{(u)}, b_{1}^{(e)}, b_{2}^{(u)}$, and $b_{2}^{(e)}$ also directly suggests that genetic and residual (co)variances and correlations between the component traits defining FE are heterogeneous across environments and management systems. What is additionally likely, but has been very difficult to infer in dairy data, is the possibility that there may be genetic variability in the coefficients $b_{1}$ and $b_{2}$ relating DMI to MilkE and to MBW for pRFI or even $b_{1}^{(u)}, b_{1}^{(e)}, b_{2}^{(u)}$, and $b_{2}^{(e)}$ for gRFI. In other words, there may be genetic variability in the conversion of DMI to MilkE and to MBW. Polygenic variation in pRFI partial regression coefficients have been inferred in other livestock species, albeit with some simplifying assumptions (Aggrey and Rekaya, 2013; Savietto et al., 2014). Based on a repeated measures analyses, Fischer et al. (2018) was able to infer cow-specific variability on partial regressions to derive RFI, but their data were not large enough to further partition these partial regressions into polygenic and permanent environmental effects. Hence, one day, selection indexes involving FE traits may involve linear combinations of the EBV of these partial regression coefficients as well.

A last comment is that the presence of heterogeneity in these partial regression coefficients will admittedly render the use of pRFI and pFS rather than gRFI or gFS to be more attractive due to computational expediency. For example, Equation [1] used to derive pRFI can be readily extended using conventional random regression methodology to allow for heterogeneity in $b_{1}, b_{2}$, and $b_{3}$ as per Tempelman et al. (2015), whereas modeling such heterogeneity for genetic and residual partial regression coefficients for gRFI requires the use of computationally intensive Bayesian methods ( $\mathrm{Lu}$ et al., 2017). Furthermore, the presence of such heterogeneity between the key component traits somewhat nullifies the use of contrast matrices like $\mathbf{K}_{u}$ to derive genetic covariances and correlations between various FE traits based on a multiple-trait genetic evaluation of the 3 key component traits of FE. Nevertheless, it should reasonably help characterize average genetic covariances and correlations between these traits. 


\section{Incorporation of Commercial Data}

Thus far, reference populations established for the genetic analysis of FE traits have been primarily limited to the use of research herds. Realizing that reliabilities of these traits are generally low due to moderate heritabilities and small reference research populations, given that DMI recording is expensive, it seems imperative to determine new strategies to incorporate data from commercial dairy operations. These strategies may include to develop and adopt data analysis methods to allow sparse recording of DMI (Negussie et al., 2019) or grouped data that comes from pens of animals ( $\mathrm{Su}$ et al., 2018) to incorporate into genetic evaluation systems.

Given that the use of sexed semen has afforded beef production to become a greater component of dairy herds (Holden and Butler, 2018), there may be opportunities to use information on feed intakes and FE on young animals as genetically correlated traits with feed intakes and $\mathrm{FE}$ in lactating cows. In fact, a sizeable component of the reference population used to provide genomic evaluations for FS for milk production in Australia is based on the use of heifer calf RFI (Pryce et al., 2015). Whether individual heifer or sibling bull calf feed intakes are easier to collect relative to lactating cow intakes is subject to debate.

Correlated proxy traits based on sensor information may facilitate incorporation of commercial data. As many commercial operations use DHI recording, milk spectral data should be already routinely available given that this data is routinely used to provide fat and protein component tests among other DHI services (Soyeurt et al., 2010). Recently, milk spectral data has been used to predict feed intake as well (Dorea et al., 2018, Wallén et al., 2018). In a much broader sense, sensor information is becoming increasingly available on large dairy herds to provide information on body temperatures, activity, and rumination activity (Gengler, 2019), for example. New promising work on image processing (Lassen et al., 2018) of 3-dimensional overhead images of cows at the feed bunk appear to be promising predictors of feed intake

One potentially intriguing aspect of the gradual availability in data on novel traits such as activity or body temperature is whether or not they should be included as energy sinks to derive RFI. For example, it has been suggested that activity or locomotion may account for 3 to $4 \%$ of energy expenditure (Berry and Crowley, 2013). In fact, as more energy sinks beyond MilkE, MBW, and $\triangle \mathrm{BW}$ are accounted for, the variability in gRFI or pRFI should naturally decrease. In some respects, these augmented definitions of RFI may be helpful in inferring genes that might be responsible for metabolic efficiency in GWA studies. However, as suggested earlier, various FE traits already involve energy sink adjustments of various degrees (e.g., FS vs. RFI) such that any sensor-trait based improvements in the prediction of DMI, FS, or even the more conventional definition of RFI (i.e., based on specifying MilkE, $\mathrm{MBW}$, and $\triangle \mathrm{BW}$ as the only energy sinks) would only help increase the reliabilities of selection indexes that include FE.

\section{Reassessing GWA Studies for RFI}

Several studies, including Li et al. (2019) and other studies cited therein, have looked at GWA involving RFI or DMI. As has been true with many GWA studies involving many other traits and species in the past, these studies have consistently not found the same associations, particularly for RFI. This is likely because of the different populations studied, the different analysis methods used, chip density, and that RFI may be simply a highly polygenic trait (Li et al., 2019).

A very popular strategy for inferring GWA analysis is based on method originally developed by Kang et al. (2010), adapted for multiple trait GWA by our group (Lu et al., 2018), and now popularized for genomic BLUP (GBLUP) in blupf90 software (Aguilar et al., 2019). The GWA inferences using this computationally tractable strategy is based on backsolving for SNP effect estimates from the genomic EBV as per Equation $[26]$.

$$
\hat{\mathbf{g}}=\mathbf{Z}^{\prime}(\mathbf{Z Z})^{-1} \hat{\mathbf{u}}_{(\text {genotyped })} .
$$

Here $\hat{\mathbf{g}}=\{\hat{g}\}_{j=1}^{m}$ is a $m \times 1$ vector of estimated SNP marker effects with $\mathbf{Z Z}^{\prime}$ as the genotypic relationship matrix between genotyped animals, $\mathbf{Z}$ being the standardized genotypes per Lu et al. (2018), and $\hat{\mathbf{u}}_{\text {(genotyped) }}$ being the EBV for genotyped animals for the trait of interest within a single-step genomic analysis that allows the incorporation of feed intake phenotypes on both genotyped and nongenotyped animals (Aguilar et al., 2010; Lu et al., 2018). The population structure is accounted for when genetic relationships are specified between phenotyped animals based on either genotype (when available) or pedigree information.

Now given that

$$
\hat{u}_{D M I, i}=b_{1}^{(u)} \hat{u}_{M i l k E, i}+b_{2}^{(u)} \hat{u}_{M B W, i}+\hat{u}_{g R F I, i}
$$

for any cow $i$, the use of GBLUP and Equation [26] implies that these same relationships between energy source and energy sink traits are defined at the SNP 
marker level $\hat{g}_{D M I, j}=b_{1}^{(u)} \hat{g}_{M i l k E, j}+b_{2}^{(u)} \hat{g}_{M B W, j}+\hat{g}_{g R F I, j}$ for any genetic marker $j$. This potentially has serious implications for GWA studies, as previously noted by Lu et al. (2018) since this expression implicitly assumes that pleiotropic effects (i.e., $b_{1}^{(u)}$ and $b_{2}^{(u)}$ ) are uniform throughout the genome, which is a rather tenuous assumption. It is not surprising then that GWA involving certain genomic regions for DMI are driven by GWA inferences on MilkE and MBW within some of those same regions when using the GBLUP assumptions as further illustrated by Lu et al. (2018).

Instead of drawing a common pleiotropic relationship across the genome as it pertains to various $\mathrm{FE}$ derivative traits and their component traits, more flexible parameterizations and modeling should be sought. Bayesian multivariate GWA techniques allowing for variable selection seem to be particularly promising in this regard (Cheng et al., 2018; Karaman et al., 2018).

\section{CONCLUSIONS}

We have striven to demonstrate extending arguments previously made by Kennedy et al. (1993) that it is inconsequential whether DMI, RFI, FS, or RME is included alongside energy sink traits like MilkE and MBW in a selection index. This is in spite of the fact that the heritability of these FE traits and their genetic correlations with other traits can vary substantially from each other. Choosing a FE trait for EBV reporting would matter if breeders wished to deviate from selecting on the index and target something closer to metabolic efficiency such as RFI or something closer to economic efficiency such as FS or RME. Of the various FE traits, however, FS might be the most meaningful for targeted selection and hence for incorporation within an index. Nevertheless, we believe that RFI is still most relevant for metabolic efficiency studies ( $\mathrm{Lu}$ et al., 2018) as FS is a genetically complex composite trait that involves both metabolic efficiency and BW.

We further advocate the use of genetic regressions in deriving FE traits like RFI or FS given the potentially large differences in genetic versus residual relationships between DMI and the energy sink traits. We recognize that current reliabilities for sire selection are rather low. If that reality continues for the foreseeable future, farmers should be encouraged to invest in teams of sires having individually low reliabilities to help protect against that uncertainty (Pryce et al., 2018). Also, it seems important that emerging reference populations include close relatives of top net merit bulls to help improve their reliabilities (Van Raden et al., 2018b).

There has been some indirect evidence that selection for RFI may be antagonistic to health and welfare in terms of early lactation metabolic changes (Dechow et al., 2017) and could further compromise energy balance and subsequent reproductive performance (Hurley et al., 2017). Hence, more studies to infer the genetic correlation between DMI and these traits are badly needed to more reliably incorporate DMI into selection indexes.

\section{ACKNOWLEDGMENTS}

The authors gratefully acknowledge funding from the USDA National Institute of Food and Agriculture (Washington, DC; grant numbers 2011-68004-30340 and 2011-67015-30338), the Council of Dairy Cattle Breeding (Bowie, MD), and the Foundation for Food and Agriculture Research (Washington, DC). The authors have not stated any conflicts of interest.

\section{REFERENCES}

Aggrey, S. E., and R. Rekaya. 2013. Dissection of Koch's residual feed intake: Implications for selection. Poult. Sci. 92:2600-2605. https:/ /doi.org/10.3382/ps.2013-03302.

Aguilar, I., A. Legarra, F. Cardoso, Y. Masuda, D. Lourenco, and I. Misztal. 2019. Frequentist p-values for large-scale-single step genome-wide association, with an application to birth weight in American Angus cattle. Genet. Sel. Evol. 51:28. https://doi.org/ 10.1186/s12711-019-0469-3.

Aguilar, I., I. Misztal, D. L. Johnson, A. Legarra, S. Tsuruta, and T. J. Lawlor. 2010. Hot topic: A unified approach to utilize phenotypic, full pedigree, and genomic information for genetic evaluation of Holstein final score. J. Dairy Sci. 93:743-752. https://doi.org/10 .3168/jds.2009-2730.

Bello, N. M., J. S. Stevenson, and R. J. Tempelman. 2012. Invited review: Milk production and reproductive performance: Modern interdisciplinary insights into an enduring axiom. J. Dairy Sci. 95:5461-5475. https://doi.org/10.3168/jds.2012-5564.

Berry, D., and J. Pryce. 2014. Feed efficiency in growing and mature animals. In Proc. 10th World Congress on Genetics Applied to Livestock Production, Vancouver, Canada. ASAS, Champaign, IL.

Berry, D. P., and J. J. Crowley. 2013. Cell Biology Symposium: Genetics of feed efficiency in dairy and beef cattle. J. Anim. Sci. 91:1594-1613. https://doi.org/10.2527/jas.2012-5862.

Britt, J. H., R. A. Cushman, C. D. Dechow, H. Dobson, P. Humblot, M. F. Hutjens, G. A. Jones, P. S. Ruegg, I. M. Sheldon, and J. S. Stevenson. 2018. Invited review: Learning from the future 2014 - A vision for dairy farms and cows in 2067. J. Dairy Sci. 101:37223741. https://doi.org/10.3168/jds.2017-14025.

Cameron, N. D. 1997. Selection indices and prediction of genetic merit in animal breeding. CAB International, Wallingford, UK.

Capper, J. L., and D. E. Bauman. 2013. The role of productivity in improving the environmental sustainability of ruminant production systems. Annu. Rev. Anim. Biosci. 1:469-489. https://doi.org/10 .1146/annurev-animal-031412-103727.

Carroll, R., D. Ruppert, L. Stefanski, and C. M. Crainiceanu. 2006. Measurement Error in Nonlinear Models: A Modern Perspective. 2nd ed. Chapman and Hall, Boca Raton, FL.

Cheng, H., K. Kizilkaya, J. Zeng, D. Garrick, and R. Fernando. 2018. Genomic prediction from multiple-trait bayesian regression methods using mixture priors. Genetics 209:89-103. https://doi.org/10 .1534/genetics.118.300650.

Coleman, J., D. P. Berry, K. M. Pierce, A. Brennan, and B. Horan. 2010. Dry matter intake and feed efficiency profiles of 3 genotypes of Holstein-Friesian within pasture-based systems of milk produc- 
tion. J. Dairy Sci. 93:4318-4331. https://doi.org/10.3168/jds.2009 -2686 .

Davis, S. R., K. A. Macdonald, G. C. Waghorn, and R. J. Spelman. 2014. Residual feed intake of lactating Holstein-Friesian cows predicted from high-density genotypes and phenotyping of growing heifers. J. Dairy Sci. 97:1436-1445. https://doi.org/10.3168/jds 2013-7205.

de Haas, Y., J. E. Pryce, M. P. Calus, E. Wall, D. P. Berry, P. Lovendahl, N. Krattenmacher, F. Miglior, K. Weigel, D. Spurlock, K. A. Macdonald, B. Hulsegge, and R. F. Veerkamp. 2015. Genomic prediction of dry matter intake in dairy cattle from an international data set consisting of research herds in Europe, North America, and Australasia. J. Dairy Sci. 98:6522-6534. https://doi.org/10 $.3168 /$ jds.2014-9257.

de Haas, Y., M. Pszczola, H. Soyeurt, E. Wall, and J. Lassen. 2017. Invited review: Phenotypes to genetically reduce greenhouse gas emissions in dairying. J. Dairy Sci. 100:855-870. https://doi.org/ $10.3168 /$ jds.2016-11246.

de Souza, R. A., R. J. Tempelman, M. S. Allen, and M. J. VandeHaar. 2019. Updating predictions of dry matter intake of lactating dairy cows. J. Dairy Sci. 102:7948-7960. https://doi.org/10.3168/ jds.2018-16176.

Dechow, C. D., C. R. Baumrucker, R. M. Bruckmaier, and J. W. Blum. 2017. Blood plasma traits associated with genetic merit for feed utilization in Holstein cows. J. Dairy Sci. 100:8232-8238. https://doi.org/10.3168/jds.2016-12502.

Dorea, J. R. R., G. J. M. Rosa, K. A. Weld, and L. E. Armentano. 2018. Mining data from milk infrared spectroscopy to improve feed intake predictions in lactating dairy cows. J. Dairy Sci. 101:58785889. https://doi.org/10.3168/jds.2017-13997.

Falconer, D., and T. Mackay. 1996. Introduction to Quantitative Genetics. Longman Group, Essex, UK.

Fischer, A., N. C. Friggens, D. P. Berry, and P. Faverdin. 2018. Isolating the cow-specific part of residual energy intake in lactating dairy cows using random regressions. Animal 12:1396-1404. https: //doi.org/10.1017/S1751731117003214.

Garcia-Ruiz, A., J. Cole, P. Vanraden, G. Wiggans, F. Ruiz, and C. Van Tassell. 2016. Changes in genetic selection differentials and generation intervals in US Holstein dairy cattle as a result of genomic selection. Proc. Natl. Acad. Sci. USA 113:E3995-E4004. https://doi.org/10.1073/pnas.1519061113.

Gengler, N. 2019. Symposium review: Challenges and opportunities for evaluating and using the genetic potential of dairy cattle in the new era of sensor data from automation. J. Dairy Sci. 102:57565763. https://doi.org/10.3168/jds.2018-15711.

Gunsett, F. C. 1984. Linear index selection to improve traits defined as ratios. J. Anim. Sci. 59:1185-1193. https://doi.org/10.2527/ jas1984.5951185x.

Harder, I., E. Stamer, W. Junge, and G. Thaller. 2019. Lactation curves and model evaluation for feed intake and energy balance in dairy cows. J. Dairy Sci. 102:7204-7216. https://doi.org/10.3168/ jds.2018-15300.

Holden, S. A., and S. T. Butler. 2018. Review: Applications and benefits of sexed semen in dairy and beef herds. Animal 12:s97-s103.

Hristov, A. N., A. T. Degaetano, C. A. Rotz, E. Hoberg, R. H. Skinner, T. Felix, H. Li, P. H. Patterson, G. Roth, M. Hall, T. L. Ott, L. H. Baumgard, W. Staniar, R. M. Hulet, C. J. Dell, A. F. Brito, and D. Y. Hollinger. 2018. Climate change effects on livestock in the Northeast US and strategies for adaptation. Clim. Change 146:33-45. https://doi.org/10.1007/s10584-017-2023-z.

Hurley, A. M., N. Lopez-Villalobos, S. McParland, E. Lewis, E. Kennedy, M. O'Donovan, J. L. Burke, and D. P. Berry. 2017. Genetics of alternative definitions of feed efficiency in grazing lactating dairy cows. J. Dairy Sci. 100:5501-5514. https://doi.org/10.3168/ jds.2016-12314.

Kang, H. M., J. H. Sul, S. K. Service, N. A. Zaitlen, S. Y. Kong, N. B. Freimer, C. Sabatti, and E. Eskin. 2010. Variance component model to account for sample structure in genome-wide association studies. Nat. Genet. 42:348-354. https://doi.org/10.1038/ng.548.

Karaman, E., M. S. Lund, M. T. Anche, L. Janss, and G. Su. 2018. Genomic prediction using multi-trait weighted GBLUP accounting for heterogeneous variances and covariances across the genome. G3: Genes|Genomes|Genetics 8:3549-3558.

Kennedy, B. W., J. H. J. Vanderwerf, and T. H. E. Meuwissen. 1993. Genetic and statistical properties of residual feed intake. J. Anim. Sci. 71:3239-3250. https://doi.org/10.2527/1993.71123239x.

Koch, R. M., L. A. Swiger, D. Chambers, and K. E. Gregory. 1963. Efficiency of feed use in beef cattle. J. Anim. Sci. 22:486-494. https: //doi.org/10.2527/jas1963.222486x.

Krattenmacher, N., G. Thaller, and J. Tetens. 2019. Analysis of the genetic architecture of energy balance and its major determinants dry matter intake and energy-corrected milk yield in primiparous Holstein cows. J. Dairy Sci. 102:3241-3253. https://doi.org/10 $.3168 /$ jds.2018-15480.

Lassen, J., J. R. Thomasen, R. H. Hansen, G. G. B. Nielsen, E. Olsen, P. R. B. Stentebjerg, N. W. Hansen, and S. Borchersen. 2018. Individual measures of feed intake on in-house commercial dairy cattle using 3D camera technology. Page 635 in Proc. World Congress on Genetics Applied to Livestock Production, Auckland, New Zealand. http://www.wcgalp.org/proceedings/2018.

Li, B., L. Fang, D. J. Null, J. L. Hutchison, E. E. Connor, P. M. VanRaden, M. J. VandeHaar, R. J. Tempelman, K. A. Weigel, and J. B. Cole. 2019. High-density genome-wide association study for residual feed intake in Holstein dairy cattle. J. Dairy Sci. 102:1106711080. https://doi.org/10.3168/jds.2019-16645.

Lidauer, M. H., A.-M. Leino, S. Stephansen, J. Poso, U. S. Nielsen, W. F. Fikse, and G. P. Aamand. 2019. Genetic evaluation for maintenance - towards genomic breeding values for saved feed in Nordic dairy cattle. Interbull Bull. 55:21-25.

Lu, Y., M. J. VandeHaar, D. M. Spurlock, K. A. W. Eigel, L. E. Armentano, C. R. Staples, E. E. Connor, Z. Wang, M. Coffey, R. F. Veerkamp, Y. de Haas, and R. J. Tempelman. 2017. Modeling genetic and nongenetic variation of feed efficiency and its partial relationships between component traits as a function of management and environmental factors. J. Dairy Sci. 100:412-427. https: //doi.org/10.3168/jds.2016-11491.

Lu, Y., M. J. Vandehaar, D. M. Spurlock, K. A. Weigel, L. E. Armentano, E. E. Connor, M. Coffey, R. F. Veerkamp, Y. de Haas, C. R. Staples, Z. Wang, M. D. Hanigan, and R. J. Tempelman. 2018. Genome-wide association analyses based on a multiple-trait approach for modeling feed efficiency. J. Dairy Sci. 101:3140-3154. https://doi.org/10.3168/jds.2017-13364.

Lu, Y., M. J. Vandehaar, D. M. Spurlock, K. A. Weigel, L. E. Armentano, C. R. Staples, E. E. Connor, Z. Wang, N. M. Bello, and R. J. Tempelman. 2015. An alternative approach to modeling genetic merit of feed efficiency in dairy cattle. J. Dairy Sci. 98:6535-6551. https://doi.org/10.3168/jds.2015-9414.

Manzanilla-Pech, C. I., R. F. Veerkamp, R. J. Tempelman, M. L. van Pelt, K. A. Weigel, M. VandeHaar, T. J. Lawlor, D. M. Spurlock, L. E. Armentano, C. R. Staples, M. Hanigan, and Y. De Haas. 2016. Genetic parameters between feed-intake-related traits and conformation in 2 separate dairy populations-the Netherlands and United States. J. Dairy Sci. 99:443-457. https://doi.org/10.3168/ jds.2015-9727.

Moraes, L. E., E. Kebreab, A. B. Strathe, J. Dijkstra, J. France, D. P. Casper, and J. G. Fadel. 2015. Multivariate and univariate analysis of energy balance data from lactating dairy cows. J. Dairy Sci 98:4012-4029. https://doi.org/10.3168/jds.2014-8995.

Negussie, E., T. Mehtiö, P. Mäntysaari, P. Løvendahl, E. A. Mäntysaari, and M. H. Lidauer. 2019. Reliability of breeding values for feed intake and feed efficiency traits in dairy cattle: When dry matter intake recordings are sparse under different scenarios. J. Dairy Sci 102:7248-7262. https://doi.org/10.3168/jds.2018-16020.

NRC. 2001. Nutrient Requirements of Dairy Cattle. 7th rev. ed. Natl. Acad. Press, Washington, DC.

Pellikaan, F. 2018. Save on feed not used for milk production. Pages 6-9 in Veeteelt. Arnhem, the Netherlands.

Pryce, J. E., O. Gonzalez-Recio, G. Nieuwhof, W. J. Wales, M. P. Coffey, B. J. Hayes, and M. E. Goddard. 2015. Hot topic: Definition and implementation of a breeding value for feed efficiency in dairy cows. J. Dairy Sci. 98:7340-7350. https://doi.org/10.3168/ jds.2015-9621. 
Pryce, J. E., T. T. T. Nguyen, M. Axford, G. Nieuwhof, and M. Shaffer. 2018. Symposium review: Building a better cow-The Australian experience and future perspectives. J. Dairy Sci. 101:37023713. https://doi.org/10.3168/jds.2017-13377.

Quaas, R. L. 1976. Computing diagonal elements and inverse of a large numerator relationship matrix. Biometrics 32:949-953. https://doi .org $/ 10.2307 / 2529279$.

Robinson, W. S. 1950. Ecological correlations and the behavior of individuals. Am. Sociol. Rev. 15:351-357. https://doi.org/10.2307/ 2087176.

Savietto, D., D. P. Berry, and N. C. Friggens. 2014. Towards an improved estimation of the biological components of residual feed intake in growing cattle. J. Anim. Sci. 92:467-476. https://doi.org/ $10.2527 /$ jas.2013-6894

Seymour, D. J., A. Canovas, C. F. Baes, T. C. S. Chud, V. R. Osborne, J. P. Cant, L. F. Brito, B. Gredler-Grandl, R. Finocchiaro, R. F. Veerkamp, Y. de Haas, and F. Miglior. 2019. Invited review: Determination of large-scale individual dry matter intake phenotypes in dairy cattle. J. Dairy Sci. 102:7655-7663. https://doi.org/ $10.3168 /$ jds.2019-16454.

Shirali, M., P. F. Varley, and J. Jensen. 2018. Bayesian estimation of direct and correlated responses to selection on linear or ratio expressions of feed efficiency in pigs. Genet. Sel. Evol. 50:33. https:/ /doi.org/10.1186/s12711-018-0403-0.

Soyeurt, H., I. Misztal, and N. Gengler. 2010. Genetic variability of milk components based on mid-infrared spectral data. J. Dairy Sci. 93:1722-1728. https://doi.org/10.3168/jds.2009-2614.

Su, G., P. Madsen, B. Nielsen, T. Ostersen, M. Shirali, J. Jensen, and O. F. Christensen. 2018. Estimation of variance components and prediction of breeding values based on group records from varying group sizes. Genet. Sel. Evol. 50:42. https://doi.org/10.1186/ s12711-018-0413-y.

Tempelman, R. J., D. M. Spurlock, M. Coffey, R. F. Veerkamp, L. E. Armentano, K. A. Weigel, Y. de Haas, C. R. Staples, E. E. Connor, Y. Lu, and M. J. VandeHaar. 2015. Heterogeneity in genetic and nongenetic variation and energy sink relationships for residual feed intake across research stations and countries. J. Dairy Sci 98:2013-2026. https://doi.org/10.3168/jds.2014.8510.

Van Raden, P. M., J. B. Cole, and K. L. Parker Gaddis. 2018a. Net merit as a measure of lifetime profit: 2018 revision.

Van Raden, P. M., J. O'Connell, E. E. Connor, M. VandeHaar, R. J. Tempelman, and K. Weigel. 2018b. Including feed intake from U.S.
Holsteins in genomic prediction. Page 125 in Proc. World Congress on Genetics Applied to Livestock Production, Auckland, NZ. http: //www.wcgalp.org/proceedings/2018.

VandeHaar, M. J., L. E. Armentano, K. Weigel, D. M. Spurlock, R. J. Tempelman, and R. Veerkamp. 2016. Harnessing the genetics of the modern dairy cow to continue improvements in feed efficiency. J. Dairy Sci. 99:4941-4954. https://doi.org/10.3168/jds .2015-10352.

VandeHaar, M. J., and R. J. Tempelman. 2016. Feeding and breeding to improve feed efficiency and sustainability. Pages 61-68 in Large Dairy Herd Management. 3rd ed. D. K. Beede, ed. American Dairy Science Association, Champaign, IL.

Veerkamp, R. F. 1998. Selection for economic efficiency of dairy cattle using information on live weight and feed intake: a review. J. Dairy Sci. 81:1109-1119. https://doi.org/10.3168/jds.S0022 -0302(98)75673-5.

Veerkamp, R. F., G. C. Emmans, A. R. Cromie, and G. Simm. 1995. Variance components for residual feed intake in dairy cows. Livest. Prod. Sci. 41:111-120. https://doi.org/10.1016/0301 -6226(94)00056-D.

Veerkamp, R. L., J. E. Pryce, D. M. Spurlock, D. P. Berry, M. P. Coffey, P. Lovendahl, R. van der Linde, J. Bryant, F. Miglior, Z. Wang, M. Winters, N. Krattenmacher, N. Charfeddine, J. Pedersen, and Y. de Haas. 2013. Selection on feed intake or feed efficiency: A position paper from gDMI breeding goal discussions. Pages 15-22 in Proc. Interbull Annual Meetings. Vol. Interbull No. 47. Nantes, France.

Wagner, C. H. 1982. Simpson's paradox in real life. Am. Stat. 36:46-48.

Wallén, S. E., E. Prestlokken, T. H. E. Meuwissen, S. McParland, and D. P. Berry. 2018. Milk mid-infrared spectral data as a tool to predict feed intake in lactating Norwegian Red dairy cows. J. Dairy Sci. 101:6232-6243. https://doi.org/10.3168/jds.2017-13874.

Zetouni, L., M. Henryon, M. Kargo, and J. Lassen. 2017. Direct multitrait selection realizes the highest genetic response for ratio traits. J. Anim. Sci. 95:1921. https://doi.org/10.2527/jas2016.1324.

\section{ORCIDS}

R. J. Tempelman @ https://orcid.org/0000-0002-7833-6730 